\title{
HYBRID DETERMINISTIC STOCHASTIC SYSTEMS WITH MICROSCOPIC LOOK-AHEAD DYNAMICS*
}

\author{
M.A. KATSOULAKIS ${ }^{\dagger}$, A.J. MAJDA ${ }^{\ddagger}$ AND A. SOPASAKIS $§$
}

Dedicated to the sixtieth birthday of Professor Andrew Majda

\begin{abstract}
We study the impact of stochastic mechanisms on a coupled hybrid system consisting of a general advection-diffusion-reaction partial differential equation and a spatially distributed stochastic lattice noise model. The stochastic dynamics include both spin-flip and spin-exchange type interparticle interactions. Furthermore, we consider a new, asymmetric, single exclusion process, studied elsewhere in the context of traffic flow modeling, with an one-sided interaction potential which imposes advective trends on the stochastic dynamics. This look-ahead stochastic mechanism is responsible for rich nonlinear behavior in solutions. Our approach relies heavily on first deriving approximate differential mesoscopic equations. These approximations become exact either in the long range, Kac interaction partial differential equation case, or, given sufficient time separation conditions, between the partial differential equation and the stochastic model giving rise to a stochastic averaging partial differential equation. Although these approximations can in some cases be crude, they can still give a first indication, via linearized stability analysis, of the interesting regimes for the stochastic model. Motivated by this linearized stability analysis we choose particular regimes where interacting nonlinear stochastic waves are responsible for phenomena such as random switching, convective instability, and metastability, all driven by stochasticity. Numerical kinetic Monte Carlo simulations of the coarse grained hybrid system are implemented to assist in producing solutions and understanding their behavior.
\end{abstract}

Key words. Coupled hybrid systems, stochastic closures, multiscale interactions, look-ahead dynamics, critical phenomena, Monte Carlo. Methods;

AMS subject classifications. 34E13, 82C27, 93C15, 37M20, 65C05.

\section{Introduction}

Hybrid systems, consisting of coupled deterministic partial differential equations and microscopic, stochastic lattice particle systems, arise in numerous applied fields $[23,15,20,18,24,14]$ and can exhibit a host of complex behaviors. In this work we study the influence of micro-/sub-grid scale fluctuations in hybrid dynamical systems and microscopic, stochastic lattice particle systems. We focus on model prototype systems similar to those proposed in [6] that are computationally tractable with direct numerical simulations but can still exhibit a host of complex phenomena $[6,7,19]$. Microscopic noise will significantly alter the deterministic mean field behavior of the hybrid system yielding corresponding regimes with phenomena driven by the interaction of nonlinearity and noise across scales, such as strong intermittency, stochastic resonance, and random oscillations. Our findings $[6,7,8]$ strongly suggest that deterministic closures of the hybrid system such as mean field, a stochastic averaging principle, or moment equations may be either inadequate as descriptions of the overall system or simply difficult to both derive and assess their effectiveness.

In general fluctuations play a dominant role in the stochastic system as is evident in long time simulations [7] and asymptotic analysis in a linearized stochastic PDE

\footnotetext{
*Received: December 2, 2008; accepted (in revised version): March 19, 2009.

$\dagger$ Department of Mathematics and Statistics, University of Massachusetts, Amherst, MA 01003 , USA (markos@math.umass.edu).

${ }^{\ddagger}$ Courant Institute of Mathematical Sciences and Center for Atmospheric and Ocean Sciences, New York University, New York, NY 10012, USA (jonjon@cims.nyu.edu).

$\S$ Department of Mathematics and Statistics, University of North Carolina at Charlotte, Charlotte, NC 28223, USA (asopasak@uncc.edu).
} 
limit [8]. The influence of fluctuations is also pronounced in the case of phase transition regimes. In general we show that fluctuation-driven rare events occur in several parameter regimes and are not captured by the deterministic mesoscopic equations. Along these lines, computational closure methods relying on relatively short runs of microsimulators such as the equation-free [2, 13] and HMM [4] approaches may also have to account for such phenomena when deciding the integration time for the micro simulation and determining suitable coarse-grained observables such as a number of moments.

We face challenges both in implementation and simulation, as well as in the interpretation of the results of such hybrid systems. Problems arise in the direct numerical simulation of realistic size systems due to scale disparities between the discrete stochastic microscopic models and the continuum macroscopic equations. Secondly, the fact that the coupled systems have nonlinear interactions across a wide range of scales implies that the stochasticity inherited from the microscopic model can play a subtle but important role in the dynamic behavior of the overall system. There is a critical interplay between interactions across scales and random fluctuations which must be kept into consideration in any type of closure. These considerations motivated work in $[6,7]$ as well as in the present paper, advocating methods capable of producing solutions in reasonable time while mainting some control of the possible error committed while still capturing the essential features of those solutions. We consider a class of stochastic closures that yield a computationally efficient hybrid system while still capturing the transient and long-time behavior of the full hybrid system. Such stochastic closures were first developed in [7] for hybrid systems without phase transitions in the microscopic dynamics, as well as in [14] as a stochastic parameterization of unresolved features of tropical convection and are based on systematic coarse-grainings of (uncoupled) stochastic lattice dynamics that were developed in $[9,10,12]$. The goal there was to describe microscopic stochastic lattice dynamics with a hierarchy of coarser - and thus computationally preferable — but still discrete, stochastic observables, while maintaining a controlled error in the approximation. The related stochastic closures for hybrid systems with phase transitions, presented in section 2.3, give rise to computationally inexpensive reduced hybrid models that correctly capture the transient and long-time behavior of the full hybrid system. Such closures capture essential features of their complex hybrid counterparts but allow for testing computationally inexpensive mesoscopic deterministic and stochastic closures and permit computationally feasible detailed statistical comparisons of the derived mesoscopic theories against direct numerical simulations of the full hybrid system. We further enhance the stochastic dynamics and introduce a new type of stochastic look-ahead interactions with direct links to physical applications $[21,22,1]$. As a result the hybrid system under the influence of directional fluctuations from the stochastic piece may cooperate or conflict against advective trends arising from the PDE. New solution profiles emerge which can not be predicted in terms of stability analysis. Stochastically induced advective effects and their impact on these solutions are analyzed.

This paper has the following outline. We introduce the hybrid system in section 2.1. We then provide all the details for the microscopic stochastic surface processes in section 2.2. In particular we present details about stochastic processes for adsorption/desorption and/or surface diffusion of particles with spin-flip/exchange Ising systems coupled to a PDE exhibiting a similar variety of dynamic and stability behaviors in different parameter regimes. Both microscopic and coarse grained dynamics are 
described in detail and coarse grained approximations are produced in that section. Subsequently stochastic averaging closures as well as mesoscopic approximations for symmetric and look-ahead potentials are obtained in section 3. These approximations are then subjected to linear stability analysis in section 4 . In that section we are able to identify parameter regimes which are critical toward stability of solutions. These parameters are subsequently perturbed through simulations producing numerical solutions for our hybrid system in section 5 .

\section{System dynamics and stochastic mechanisms}

In this section we give the details of the coupled system which is comprised of a PDE and a stochastic noise model. We pay particular attention to the structure of the stochastic component for this system. Specifically spin-flip and spin-exchange dynamics are described for both look-ahead as well as classical potentials. Although we start by describing the microscopic look-ahead dynamics in section 2.2 it is mainly the coarse grained look-ahead dynamics which we focus on in section 2.3.

2.1. System overview. We propose the following coupled system in variables $(Y, u) \equiv(Y(x, t), u(x, t))$ which represent the evolution of a deterministic partial differential equation (PDE) coupled to a spatially stochastic lattice noise model

$$
\left\{\begin{aligned}
\partial_{t} Y & =\frac{1}{\tau_{c}} \mathcal{F}[Y, u]:=\frac{1}{\tau_{c}}\left(D \partial_{x}^{2} Y+A \partial_{x} Y+R Y+M u+C\right) \\
\mathbf{E} \partial_{t} f(\sigma) & =\mathbf{E} \mathcal{L} f(\sigma) .
\end{aligned}\right.
$$

Here $\mathbf{E}$ denotes expected value, $f$ a test function, $\sigma(x, t)$ denotes a microscopic stochastic Ising type process, details of which will be provided below, and evolves on a fine lattice $\Lambda=\{1,2, \ldots, N\}$ comprised of $N$ cells. The microscopic generator $\mathcal{L}$ incorporates both spin-flip and spin-exchange dynamics

$$
\mathcal{L}=d_{f} \mathcal{L}_{s f}+d_{e} \mathcal{L}_{s e}
$$

and is described in detail in section 3 . The coefficients $d_{f}$ and $d_{e}$ involve the characteristic time for the corresponding stochastic process. The parameter $\tau_{c}$ appearing in (2.1), on the other hand, denotes the characteristic time for the deterministic equation. The quantity $u=\bar{\sigma}$ ranges as $0 \leq u \leq 1$ and represents the averaged solution over a spatial subdomain. Many instances of the constants $D, A, R, M, C$ will be specified later, in the numerics of section 5 , according to behavior desired to be exhibited by the PDE (excitable, oscillatory, etc.). Note that the coupling mechanism for system (2.1) is bidirectional. The PDE is coupled to the stochastic model through the variable $u(x, t)$, a local density quantity, while the stochastic model is coupled to the PDE through an external interaction potential function $h(Y)$ (see section 2.2.2). In this work we derive and analyze a coarse grained approximation of (2.1) which will be used in order to obtain our main results. Error estimates as well as potential impact and limitations of this coarse grained approximation have been established in [11]. The coarse grained equivalent of (2.1) is realized as follows: we introduce a new coarse lattice $\Lambda_{M}$ on which this new coarse process will be defined. We therefore define $M$ coarse cells $D_{k}, k=1, \ldots, M$, and $N=M q$ based on the microscopic number of cells $N$ of the original lattice $\Lambda$. We define a new Markovian process $\eta(k, t)$ as an averaged quantity over each coarse cell $D_{k}$

$$
u(k, t)=\eta(k, t):=\frac{1}{q} \sum_{x \in D_{k}} \sigma(x, t) .
$$




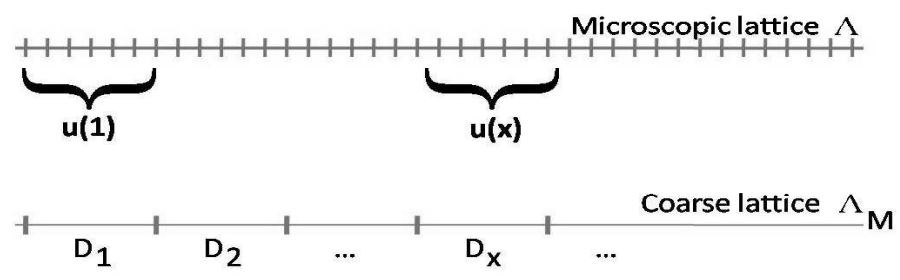

FIG. 2.1. Lattice coarse graining

See figure 2.1 as well as section 2.3 for the details.

$$
\left\{\begin{aligned}
\partial_{t} Y & =\frac{1}{\tau_{c}} \mathcal{F}[Y, u]:=\frac{1}{\tau_{c}}\left(D \partial_{x}^{2} Y+A \partial_{x} Y+R Y+M u+C\right) \\
\mathbf{E} \partial_{t} f(\eta) & =\mathbf{E} \mathcal{L}^{C} f(\eta)
\end{aligned}\right.
$$

where the coarse grained generator $\mathcal{L}^{C}$ in $(2.2)$ is an approximation of the original microscopic generator $\mathcal{L}$ in (2.1). More details for the coarse grained approximation $\eta$, its generator $\mathcal{L}^{C}$, and in general the coarse grained coupled system will be provided in section 2.3 .

2.2. Microscopic dynamics. We start by describing the stochastic microscopic spin dynamics. We define an one-dimensional periodic lattice which is partitioned into $N$ cells via,

$$
\Lambda=\{1,2, \ldots, N\} .
$$

On each of the lattice points $x \in \Lambda$ we define an order parameter $\sigma(x)$ via,

$$
\sigma(x)=\left\{\begin{array}{l}
1, \text { if there exists a particle at site } x \\
0, \text { if the site at } x \text { is empty. }
\end{array} .\right.
$$

A spin configuration $\sigma$ is an element of the configuration space $\Sigma=\{0,1\}^{\Lambda}$ and we write $\sigma=\{\sigma(x): x \in \Lambda\}$ where $\sigma(x)$ denotes the spin at location $x$. Since there are $|\Lambda|$ sites on the lattice then the system can be in any of $2^{|\Lambda|}$ possible states. The local situation at each of those states is appraised by the interaction potential $J$. We let $J$ denote, as usual, a symmetric short range interaction potential,

$$
J(x, y)=\gamma V(\gamma|x-y|), x, y \in \Lambda
$$

where $\gamma=1 /(2 L+1)$ is a parameter prescribing the range of microscopic interactions and therefore $L$ denotes the potential radius. Here we let $V: R \rightarrow R$ and set,

$$
V(r)=0, r \in R^{-} \text {and } V(r)=0 \text { for } r \geq 1 .
$$

For now we assume a uniform potential of the form,

$$
V(r)= \begin{cases}J_{0}, & \text { if } 0<r<1 \\ 0, & \text { otherwise }\end{cases}
$$

where $J_{0}$ is a parameter which describes attractive, repulsive, or no-interactions based on its sign. 
2.2.1. ASEP with look-ahead potential. In models involving stochastic lattice dynamics it is common to employ a symmetric interaction potential $J$ conceptually similar in form to the one presented in (2.4). Micromagnetic interactions is an example of a typical application where a symmetric interaction potential works well to simulate the physical properties of magnetic dipoles. Here we propose a different type of potential which, due to a novel direction mechanism, attributes advective affects in the stochastic dynamics. This one-sided potential originated from modeling applications in traffic flow $[21,22]$ where look-ahead dynamics produce a natural medium for the physics involved in vehicle motion. We introduce this new mechanism and stochastic dynamics by focusing on the interaction potential $J$ in (2.4) which involves a simple but crucial modification,

$$
J(x, y)=\gamma V(\gamma(x-y)), \quad x, y \in \Lambda
$$

where once again $\gamma=1 /(2 L+1)$ is a parameter prescribing the range of microscopic interactions and $L$ is the potential radius and $V$ as in (2.5). Note however that now this modification of $V$ and $J$ allows direction detection since only interactions where $x>y$, are possible. This seemingly simple potential function is responsible for completely altering the behavior of our system since, as we will see later through stability analysis and numerical results in sections 4 and 5 , it creates advective trends in the stochastic component of the system (2.2).

2.2.2. Diffusion Arrhenius dynamics. In this section we describe a stochastic process equipped with look-ahead conservative, spin-exchange type, Arrhenius dynamics. Under this engine the simulation is driven based on the energy barrier a particle has to overcome in changing from one state to another. During such a spin-exchange between nearest neighbor sites $x$ and $y$ the system will actually allow the order parameter $\sigma(x)$ at location $x$ to exchange value with the one at $y$. We keep track of inter-particle interactions via the potential $U(x)$ which is comprised of contributions from short range, exchange interactions $U_{s e}$, and external interactions, $h(Y)$ :

$$
U(x)=U_{s e}(x)+h(Y) .
$$

The stochastic model is coupled to the PDE through the external potential $h(Y)$. We assume for simplicity that $h(Y)$ depends linearly on $Y$ as follows:

$$
h(Y)=\alpha(Y+\gamma)
$$

for given parameters $\alpha$ and $\gamma$. This coupling mechanism is simple yet sufficient to produce interesting departures from usual mean field behavior. Nonlinear dependence of the external potential to $Y$ is also possible and have been explored in detail $[6,8]$. The short range exchange interaction potential is described via [17]:

$$
U_{s e}(x)=\sum_{z \neq x} J_{s e}(x, z) \sigma(z)
$$

with $J_{s e}$ from (2.4). Note that the spin-exchange, due to the specific construction of the interaction potential $J_{s e}$ in $(2.4)$, can take effect if and only if the location at $x$ is occupied while the location at $y$ is not. Overall, the rate at which the stochastic process $\left\{\sigma_{t}\right\}_{t \geq 0}$ will perform a spin-exchange using Arrhenius dynamics is defined [17] 
via

$$
c_{s e}(x, y, \sigma)= \begin{cases}c_{0} \exp [-U(x)], & \text { if } \sigma(x)=1, \sigma(y)=0 \\ c_{0} \exp [-U(y)], & \text { if } \sigma(x)=0, \sigma(y)=1 \\ 0, & \text { otherwise }\end{cases}
$$

where $c_{0}=1 / \tau_{0}$ with $\tau_{0}$ the characteristic or relaxation time for the process. We note that for classical potentials $y=x \pm 1$ while for one-sided potentials, (2.9) implies that $y=x+1$. figure 2.2 displays such a situation. The stochastic process $\left\{\sigma_{t}\right\}_{t \geq 0}$, whose

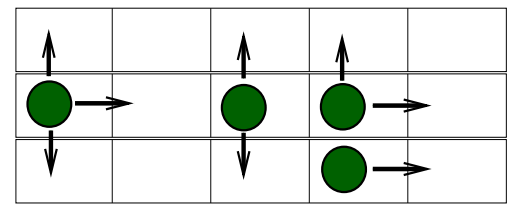

FIG. 2.2. One-sided potential interactions in a given lattice.

dynamics were described in detail in this section, is a continuous time, jump, Markov process on $L^{\infty}(\Sigma ; R)$ with generator $[17]$

$$
\left(\mathcal{L}_{s e} f\right)(\sigma)=\sum_{x, y \in \Lambda} c_{s e}(x, y, \sigma)\left[f\left(\sigma^{x, y}\right)-f(\sigma)\right]
$$

for any bounded test function $f \in L^{\infty}(\Sigma ; R)$ with $c_{s e}(x, y, \sigma)$ defined in (2.9). Here $\sigma^{x, y}$ denotes the configuration after an exchange of the spins at location $x$ and $y$ such that,

$$
\sigma^{x, y}(z)=\left\{\begin{array}{l}
\sigma(y) \text { if } z=x \\
\sigma(x) \text { if } z=y \\
\sigma(z) \text { otherwise. }
\end{array}\right.
$$

Therefore observables $f$ (test functions) evolve with the rule

$$
\frac{d}{d t} E f(\sigma)=E\left(\mathcal{L}_{s e} f\right)(\sigma)
$$

which is equivalent to the Dynkin formula.

2.2.3. Non-conservative microscopic dynamics. We now further equip our stochastic process $\{\sigma\}_{t \geq 0}$ with non-conservative, spin-flip, Arrhenius dynamics. During a spin-flip the system will actually allow the order parameter $\sigma(x)$ at location $x$ to change value without reciprocating this change at any other location. This is interpreted as placing (removing) a particle at (from) $x$ if $\sigma(x)=0(1)$. Thus the overall lattice density can now be changed according to influences from the PDE and vice versa. As a result conservation of mass on the lattice will not hold while such a process is active. On the application side, once again these dynamics allow our system to model a number of non-conservative physical processes. The rate at which a process will perform such a spin-flip Arrhenius move is given by [17]

$$
c_{s f}(x, \sigma)= \begin{cases}c_{d} e^{-\beta U_{s f}(x)}, & \text { when } \sigma(x)=1 \\ c_{a}, & \text { when } \sigma(x)=0,\end{cases}
$$


where

$$
U_{s f}(x)=\sum_{z \neq x} J_{s f}(x, z) \sigma(z)
$$

We assume for simplicity that both the adsorption and desorption constants are equal $c_{1}:=c_{a}=c_{d}$, where $c_{1}=1 / \tau_{1}$ denotes the characteristic, or relaxation, time of the spin-flip process. This together with section 2.2.2 completes the details for both spin-flip and spin-exchange microscopic stochastic dynamics which characterize the interactions in this model. Note that the mechanisms which we developed in section 2.2.2 and 2.2.3 can be realized in its full generality with two different interaction potential functions $J_{s e}$ and $J_{s f}$. For simplicity however we allow here that $J \equiv J_{s f}=$ $J_{s e}$.

REMARK 2.1. The combined mechanism for spin-flip and spin-exchange dynamics is given by aggregating together rates $(2.9)$ and (2.12). Thus the probability for spin-flip at $x$ or spin-exchange between locations $x$ and $y$ during time $[t, t+\Delta t]$ is given by $[17]$

$$
c_{s f}(x, \sigma) \Delta t+c_{s e}(x, y, \sigma) \Delta t+O\left(\Delta t^{2}\right) .
$$

2.2.4. Invariant measures. Since there are $N=|\mathcal{L}|$ sites on the lattice, the system can be in any of $2^{|\mathcal{L}|}$ possible states while the energy of any particular state is given by the following Hamiltonian,

$$
\mathcal{H}(\sigma)=-\frac{1}{2} \sum_{x \in \mathcal{L}} \sum_{y \neq x} J(x, y) \sigma(x) \sigma(y)+\sum_{x} h \sigma(x),
$$

where $J(x, y)$ is an interparticle potential as in (2.4) and $h$ is a given external potential. Here $J$ is assumed to be even, $J(r)=J(-r)$, and as an example we can take a form similar to (2.5). Applying a spin-flip stochastic model we create new states from old ones generated by a Markov process as described previously. Equilibrium states of the stochastic model are described by the Gibbs states

$$
\mu_{\beta, N}(d \sigma)=\frac{1}{Z} e^{\beta \mathcal{H}(\sigma)} P_{N}(d \sigma)
$$

where $\beta=1 /(k T)$ and $T$ denotes the temperature. Here $P_{N}(d \sigma)$ denotes the (product) prior distribution on $\mathcal{L}$,

$$
P_{N}(d \sigma)=\prod_{x \in \mathcal{L}} p(d \sigma(x)) \text { and } p(\sigma(x)=0)=\frac{1}{2}, p(\sigma(x)=1)=\frac{1}{2},
$$

where $Z$ is the partition function, guaranteeing that $(2.16)$ is a probability measure. Note that $k=1.3810^{-23} J K^{-1}$ is the Boltzmann constant. Note that in the case of a look-ahead potential such as the one presented in section 2.2.1 it is not clear whether such a Hamiltonian description is valid or can even exist at all. The stochastic dynamics described by an ASEP no longer guarantee that an invariant Gibbs state may exist. Nevertheless, for several applications it is not the equilibrium dynamics which we are after but the transient states. Therefore we undertake description of such non-equilibrium look-ahead potentials with respect to this perspective and propose these dynamics in order to describe complex physical processes. 
2.3. Coarse grained approximations. We now apply ideas from [9] and introduce a process $\eta$ on a new coarse lattice which will approximate the original microscopic process $\sigma$. This new coarse process $\eta$ will furthermore be shown to produce a stochastic closure of the original full system (2.1). Through this coarse graining it will be possible to extract a closed stochastic coupled system which does not depend on the expected value of the microscopic process $\sigma$ but instead on the new coarse process $\eta(k)$. Rigorous details for the coarse process $\eta$ as well as estimates of the approximation error can be found in [11]. We start by first defining the new coarse lattice $\Lambda_{M}$. We consider $M$ coarse cells $D_{k}$ where $1 \leq k \leq M$, each of size $q$, so that $N=M q$. We let $\Lambda_{M}=\{1,2, \ldots, M\}$ represent a new coarse lattice where $\Lambda_{M} \subset \Lambda$. We can now define the new coarse grained random process,

$$
\eta(k)=\mathcal{T}(\sigma)(k):=\sum_{y \in D_{k}} \sigma(y), \text { for } k=1, \ldots, M
$$

and denote $\eta=\left\{\eta(k): k \in \Lambda_{M}\right\}$, with $\eta(k) \in\{0,1, \ldots, q\}$ the coverage of the coarse cell $D_{k}$ for $1 \leq k \leq M$. Note that each $D_{k}$ consists of $q$ microcells and the order parameter $\eta(k)$ counts the number of particles in each such coarse cell $D_{k}$. We further define the coarse configuration space $\mathcal{H}_{M, q}=\{0,1, \ldots, q\}^{\Lambda_{M}}$. The projection operator $\mathcal{T}$ is defined implicitly by (2.17). For any test function $g \in L^{\infty}\left(\mathcal{H}_{M, q} ; R\right)$ we have

$$
f(\sigma):=g(\mathcal{T}(\sigma))=g(\eta),
$$

where $f$ is a test function in $L^{\infty}(\Lambda ; R)$. In the next section we equip this new coarse process with either spin-flip or spin-exchange non-symmetric look-ahead dynamics. The symmetric, non look-ahead, dynamics case is also provided at the end of the section.

2.3.1. Spin-exchange coarse grained dynamics with look-ahead. We have now built the coarse grained dynamics for the new process $\eta$ which was defined above in section 2.3. The dynamics in this section allow for spin-exchange interactions using a non-symmetric, look-ahead, potential. An equivalent look-ahead stochastic mechanism for microscopic spin-exchange dynamics related to traffic flow has been proposed in [21] and [22]. The corresponding coarse grained mechanism is now introduced below. Starting from the generator (2.10) of the microscopic spin-exchange process $\sigma$ defined previously we can derive a corresponding coarse grained generator for the new coarse process $\eta$ as follows:

$$
\begin{aligned}
\frac{d}{d t} E f(\sigma) & =E \sum_{x, y \in \Lambda_{N}} c_{s e}(x, y, \sigma)\left[f\left(\sigma^{x, y}\right)-f(\sigma)\right] \\
& =E \sum_{k, l \in \Lambda_{M}} \sum_{x \in D_{k}, y \in D_{l}} c(x, y, \sigma)\left[f\left(\sigma^{x, y}\right)-f(\sigma)\right],
\end{aligned}
$$

where $c_{s e}(x, y, \sigma)$ with $y=x$ denotes the microscopic diffusion rate for look-ahead dynamics,

$$
c_{s e}(x, x+1, \sigma)= \begin{cases}c_{0} \exp [-U(x)], & \text { if } \sigma(x)=1, \sigma(x+1)=0 \\ c_{0} \exp [-U(x+1)], & \text { if } \sigma(x)=0, \sigma(x+1)=1 \\ 0, & \text { otherwise, }\end{cases}
$$

which can be written as,

$$
\begin{aligned}
& c_{s e}(x, x+1, \sigma) \\
= & c_{0} \sigma(x)(1-\sigma(x+1)) \exp (-\beta U(x))+c_{0} \sigma(x+1)(1-\sigma(x)) \exp (-\beta U(x+1)) .
\end{aligned}
$$


Note that for $x \in D_{k}$ and $y=x+1 \in D_{l}$ we have

$$
\mathcal{T}\left(\sigma^{x, y}\right)=\mathcal{T}(\sigma)+\sigma(y)(1-\sigma(x))\left(\delta_{k}-\delta_{l}\right)+\sigma(x)(1-\sigma(y))\left(\delta_{l}-\delta_{k}\right),
$$

where $\delta_{k}, \delta_{l} \in \mathcal{H}_{M, q}$ denotes the configuration with a single particle at site $k, l \in \Lambda_{M}$. Thus using the above and the mapping in (2.18) the right hand side of (2.19) can be expressed in terms of the coarse grained test function $g \in L^{\infty}\left(\mathcal{H}_{M, q} ; R\right)$ as

$$
\begin{aligned}
f\left(\sigma^{x, y}\right)-f(\sigma)= & \sigma(y)(1-\sigma(x))\left[g\left(\eta+\delta_{k}-\delta_{l}\right)-g(\eta)\right] \\
& +\sigma(x)(1-\sigma(y))\left[g\left(\eta+\delta_{l}-\delta_{k}\right)-g(\eta)\right] .
\end{aligned}
$$

Thus we obtain,

$$
\begin{aligned}
\frac{d}{d t} E g(\eta)=E \sum_{k, l \in \Lambda_{M}} \sum_{x \in D_{k}, y \in D_{l}} & c_{s e}(x, y, \sigma)\left[\sigma(y)(1-\sigma(x)) g\left(\eta+\delta_{k}-\delta_{l}\right)\right. \\
& \left.+\sigma(x)(1-\sigma(y)) g\left(\eta+\delta_{l}-\delta_{k}\right)-g(\eta)\right] .
\end{aligned}
$$

With the assumption that $x, y$ are nearest neighbors we can express (2.21) through the corresponding coarse grained potential as follows:

$$
U(x)=\bar{U}(l)+O\left(\frac{q}{2 L+1}\right),
$$

where

$$
\bar{U}(k)=\sum_{\substack{l \in \Lambda_{M} \\ l \neq k}} \bar{J}_{L A}(k, l) \eta(l)+\bar{J}_{L A}(0,0)(\eta(k)-1)
$$

and for simplicity we let $h \equiv 0$ for this derivation. The coarse grained interaction potential $\bar{J}_{L A}(k, l)$ is computed as an average of pairwise interactions between microscopic spins on coarse cells $D_{k}$ and $D_{l}$,

$$
\bar{J}_{L A}(l, k)=M^{2} \int_{D_{k}} \int_{D_{l}} J(r-s) d r d s,
$$

where $J$ as in (2.6) indicates a length range for spatial interactions in the microscopic lattice level. Note that the resulting coarse grained interaction potential as defined above in (2.24) is a coarse grained look-ahead type potential. In order now to derive the corresponding coarse grained generator for our stochastic process $\eta$ we start from the microscopic spin-exchange rate in (2.21). We assume that particles are approximately independent inside the coarse cells and replace $\sigma(x), \sigma(y)$ in (2.21) everywhere by $\eta(k) / q$ and $\eta(l) / q$ respectively. Here $l=k+1$ due to the look-ahead potential. Performing simple clean-up operations it is easy to see that (2.22) becomes

$$
\frac{d}{d t} E g(\eta)=q E \sum_{k, k+1 \in \Lambda_{M}} c_{C G}(k, k+1, \eta)\left[g\left(\eta+\delta_{k+1}-\delta_{k}\right)-g(\eta)\right],
$$

where

$$
c_{C G}(k, k+1, \eta)=c_{0} \frac{\eta(k)}{q}\left(1-\frac{\eta(k+1)}{q}\right) \exp (-\beta \bar{U}(k+1))
$$

with $c_{0}$ from (2.9). The spin-exchange coarse grained generator therefore is defined to be

$$
\mathcal{L}_{s e}^{C} g(\eta):=\sum_{k, k+1 \in \Lambda_{M}} c_{C G}(k, k+1, \eta)\left[g\left(\eta+\delta_{k+1}-\delta_{k}\right)-g(\eta)\right] .
$$


2.3.2. Spin-flip coarse grained dynamics with look-ahead. Microscopic spin-flip dynamics with look-ahead interactions have recently been explored in [3] and [1] in the context of traffic flow modeling. The coarse grained spin-flip mechanism with look-ahead interactions is now presented below. Following the derivation above (see also [9]) the coarse grained generator corresponding to the microscopic spin-flip dynamics from $(2.12)$ is

$$
\mathcal{L}_{s f}^{C} g(\eta)=\sum_{y \in \Lambda_{M}} c_{a}(y, \eta)\left[g\left(\eta+\delta_{y}\right)-g(\eta)\right]+c_{d}(y, \eta)\left[g\left(\eta-\delta_{y}\right)-g(\eta)\right],
$$

for $g \in L^{\infty}\left(\mathcal{H}_{M, q} ; R\right)$ where $c_{a}(y, \eta), c_{d}(y, \eta)$ denote the coarse grained diffusive adsorption and desorption rates. Following the ideas of the derivation for the spin-exchange process presented in section 2.3.1 above we can similarly derive the expressions for the corresponding coarse grained adsorption and desorption spin-flip rates. The coarse grained adsorption rate is

$$
c_{a}(y, \eta)=c_{1}[q-\eta(y)]
$$

with $c_{1}$ from (2.12) which expresses the rate by which $\eta(y)$ is increased by 1 , up to its possible maximum $q$. Respectively the coarse grained desorption rate is,

$$
c_{d}(y, \eta)=c_{1} \eta(y) \exp (-\beta \bar{U}(y)) \exp \left(O\left(\frac{q}{2 L+1}\right)\right),
$$

with a similar form for $\bar{U}(y)$ as in (2.23). The expression above involves a known error term due to the necessary approximation from the truncation in the microscopic exponential (see [10] for details).

REMARK 2.2. it is an easy extension of the ideas from section 2.2.1 to develop symmetric, non-look-ahead, coarse grained interaction potentials. In that respect we must employ the microscopic interaction potential $U(x)$ in (2.4) and (2.5) and based on the ideas presented in both of the previous section 2.3.1 and 2.3.2 carry out equivalent expansions and approximations in order to eliminate $E \sigma$ in favor of $\eta$. Specifically, for classical interaction potentials we replace $\bar{J}_{L A}$ with $\bar{J}$ where the functional dependence on $J$ under the integral in (2.24) is instead given by (2.4). This automatically adjusts our look-ahead potential to a symmetric potential since it allows interactions in every direction. For more details we refer to [10] and [11] where the ideas for such symmetric potentials have been provided.

2.4. Simplified coarse-grained model with look-ahead dynamics and single-column spin flip mechanism. In this section we present an outline of an alternative stochastic model with very rich phenomenology which involves a version of the coarse grained dynamics described above. The model implements a number of the stochastic mechanisms which we already described previously in the coarse grained setting. We consider the coarse grained lattice and corresponding process discussed in section 2.3 equipped with spin-exchange and look-ahead dynamics for nearest neighbors. In fact to simplify this process further we assume that the lookahead interaction range is such that, in the coarse grained setting, we only interact with a single coarse cell ahead. We further equip this process with single-column, spin-flip dynamics. The dynamics for such a spin-flip process therefore do not involve any interactions with its neighbors. As a result the spin-flip process seems to act as a birth-death type process for each corresponding coarse-grained lattice cell. Note that 


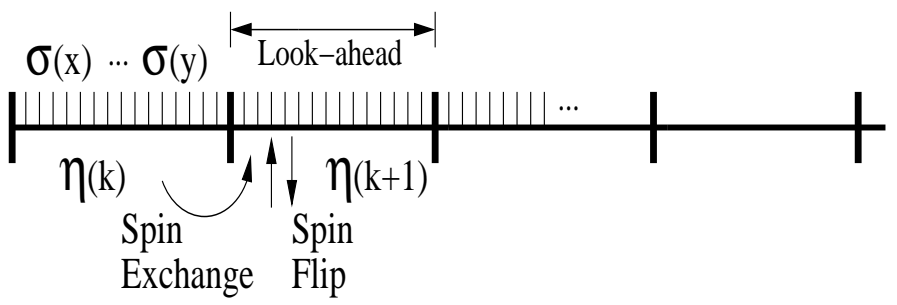

FIG. 2.3. Depiction of a simple, yet rich in behavior, coarse grained model with spin-exchange, nearest neighbor, look-ahead dynamics and birth-death type (no interactions) spin-flip dynamics. The look-ahead only encompasses one coarse grained cell.

this stochastic process is computationally inexpensive due to the overall reduction in neighbor interactions. At the same time it accounts for many basic mechanisms involving advective motion of particles as well as density changes through the spin-flip piece. Overall this simplified model is a special case of the coarse grained models of the previous section. As a result this model is simple to simulate and is expected to have similar phenomenology with the model presented previously. We will explore this model further in the future.

\section{Mesoscopic approximations}

Our perspective in this section involves obtaining mesoscopic approximations which later can be used together with linear stability analysis in order to identify critical parameter regimes responsible for nonlinear phenomena. We will present two different types of mesoscopic estimates. In the first estimate we explore the long range interaction, Kac potential, with look-ahead. In the second case we present a stochastic averaging closure where we must assume that the process is ergodic as well as that the stochastic system has a time scale separation from the deterministic piece. In this case, the long-range potential interactions requirement used for our first mesoscopic estimate is no longer necessary assuming that no phase transitions exist. We start by first obtaining closures at different temporal and spatial scales for the microscopic spin-flip, spin-exchange stochastic dynamics model. The analysis carried out here involves the case of a symmetric interaction potential as described in (2.4). The one-sided ASEP case introduced in section 2.2.1 is obtained later in section 3.2. The stochastic piece of our system is described from equation (2.11) by its generator $\mathcal{L}$, which incorporates both spin-flip and spin-exchange dynamics,

$$
\begin{aligned}
\mathcal{L} f(\sigma)= & \mathcal{L}_{s e} f(\sigma)+\mathcal{L}_{s f} f(\sigma) \\
= & \sum_{x \in \Lambda}\left(c_{1}(1-\sigma(x))+c_{1} \sigma(x) \exp \left(-U_{s f}(x, \sigma)\right)\left[f\left(\sigma^{x}\right)-f(\sigma)\right]\right. \\
& +\sum_{x \in \Lambda} c_{0} \sigma(x)(1-\sigma(x)) \exp \left(-U_{s e}(x, \sigma)\right)\left[f\left(\sigma^{x, y}\right)-f(\sigma)\right],
\end{aligned}
$$

with $c_{0}$ from (2.9) and $c_{1}$ from (2.12). Here $\sigma^{x}$ denotes the configuration prior to a change at location $x$ as follows,

$$
\sigma^{x}= \begin{cases}\sigma(y)+1=1 & \text { if } x=y \text { and } \sigma(y)=0 \\ \sigma(y)-1=0 & \text { if } x=y \text { and } \sigma(y)=1 \\ \sigma(y) & \text { otherwise, }\end{cases}
$$

and $\sigma^{x, y}$ denotes an exchange of the values of the stochastic process $\sigma$ at locations $x$ 
and $y$,

$$
\sigma^{x, y}(z)=\left\{\begin{array}{l}
\sigma(y) \text { if } z=x \\
\sigma(x) z=y \\
\sigma(z) \text { otherwise. }
\end{array} .\right.
$$

3.1. Mesoscopic equations for spin-flip/exchange dynamics. In this section we derive mesoscopic approximations based on the microscopic dynamics. More details for this procedure can be found in [9] and [10]. We let the test function $f(\sigma)=\sigma(z)$, for a specific value $z \in \Lambda$, and consider the average quantity $E \sigma_{t}(z)$. Based on (3.2) and (3.3) above we can write,

$$
\partial_{t} E \mathcal{L} \sigma(z)=\sum_{y \in n n(z)} E\left(\mathcal{L}_{s e} \sigma(y)+\mathcal{L}_{s f} \sigma(y)\right)
$$

where $n n(z)$ denotes the nearest neighbors to $z$. Thus from the above we obtain

$$
\partial_{t} E \mathcal{L} \sigma(z)=\sum_{y \in n n(z)} E\left(c_{s e}(z, y, \sigma)+c_{s f}(z, \sigma)\right) E[\sigma(y)-\sigma(z)] .
$$

Note that the above equation is not closed for $E \sigma(z)$. To close this system we pass to the limit of weak, uniform, long range interactions. At large space/time scales and for weak long range potentials the small-scale fluctuations of icing systems are suppressed and an almost deterministic pattern emerges described by (stochastic) integrodifferential equations. The limit $L^{-1} \rightarrow 0$ in the potential expression in (2.4) (i.e., long potential range) is known as the Lebowitz-Penrose limit. We further assume that our stochastic process is a perturbation of the simple exclusion process considered in [16]. As such the process has a Bernoulli product invariant measure and at local equilibrium the probability measure is expected to be a product measure. Thus we can distribute the expected value through all the products. If we further assume propagation of chaos then the fluctuations of the spins about their mean values are independent and the law of large numbers formally applies. Thus the following further approximation for both rates $c_{s f}$ and $c_{s e}$ holds,

$$
E \exp (-U(x, \sigma)) \approx \exp \left(-U(x, E(\sigma))+o_{N}(1) .\right.
$$

We now define the average density $u(z, t)=E \sigma_{t}(z)$ and drop the $o_{N}(1)$ to obtain

$$
\begin{aligned}
\partial_{t} u(z, t)= & \sum_{y \in n n(z)}\left(c_{s e}(z, y, u)+c_{s f}(z, u)\right) E[\sigma(y)-\sigma(z)] \\
= & \sum_{y \in n n(z)} c_{0} u(y, t)(1-u(z, t)) \exp \left(-\beta U_{s e}(y, u)\right) \\
& -c_{0} u(z, t)(1-u(y, t)) \exp \left(-\beta U_{s e}(z, u)\right) \\
& +c_{1}\left(1-u(z)-u(z) \exp \left(-\beta U_{s f}(z, u)\right) .\right.
\end{aligned}
$$

We now apply that $y=n n(z)$ and obtain

$$
\begin{aligned}
\partial_{t} u(z, t)= & c_{0} u(z-1)(1-u(z)) \exp \left(-\beta U_{s e}(z-1, u)\right) \\
& -c_{0} u(z)(1-u(z+1)) \exp \left(-\beta U_{s e}(z, u)\right) \\
& +c_{1}\left[1-u-u \exp \left(-\beta U_{s f}(z, u)\right)\right]+O\left(\frac{1}{N^{2}}\right) .
\end{aligned}
$$


Taylor expanding the above in $\Delta z=1 / N$ as $N \rightarrow \infty$ where $N$ denotes the number of cells in the lattice we obtain

$$
\begin{aligned}
\partial_{t} u(z, t)= & c_{0}\left(u-\frac{1}{N} u_{z}+\frac{1}{2 N^{2}} u_{z z}\right)(1-u)\left(e^{-\beta U_{s e}}+\frac{\beta}{N} U_{s e}^{\prime} e^{-\beta U_{s e}}-\frac{\beta}{2 N^{2}} U_{s e}^{\prime \prime} e^{-\beta U_{s e}}\right) \\
& -c_{0} u\left(1-u-\frac{1}{N} u_{z}-\frac{1}{2 N^{2}} u_{z z}\right) e^{-\beta U_{s e}} \\
& +c_{1}\left[1-u-u e^{-\beta U_{s f}}\right]+O\left(\frac{1}{N^{3}}\right) .
\end{aligned}
$$

The above simplifies,

$$
\begin{aligned}
\partial_{t} u(z, t)= & c_{0} e^{-\beta U_{s e}}\left[\frac{1}{N}\left(-u_{z}+2 u u_{z}+\beta U_{s e}^{\prime}(1-u) u\right)\right. \\
& \left.\frac{1}{N^{2}}\left(\frac{1}{2} u_{z z}-\beta u_{z}(1-u) U_{s e}^{\prime}-\frac{1}{2} \beta u(1-u) U_{s e}^{\prime \prime}\right)\right] \\
& +c_{1}\left[1-u-u e^{-\beta U_{s f}}\right]+O\left(\frac{1}{N^{3}}\right) .
\end{aligned}
$$

where we assume for now a symmetric type interaction potential such as (2.4),

$$
U\left(u(z)=\beta J * u(z):=\int_{-\infty}^{\infty} J(y-z) u(y) d y .\right.
$$

Here $J$ can be either $J_{s e}$ or $J_{s f}$. In the limit of $N \rightarrow \infty$ we omit the $O\left(\frac{1}{N^{3}}\right)$ term in (3.7) and obtain,

$$
\partial_{t} u(z, t)+c_{0} \frac{1}{N} \partial_{z} F_{*}(z, t)+c_{0} \frac{1}{N^{2}} \partial_{z} H_{*}(z, t)=c_{1} G_{*}(z, t),
$$

where $F_{*}(z), G_{*}(z)$, and $H_{*}(z)$ are as follows:

$$
\begin{aligned}
& F_{*}(z, t):=u(z)(1-u(z)) \exp \left(-\beta J_{s e} * u(z)+h(z)\right), \text { and } \\
& H_{*}(z, t):=\left[u_{z}-\beta u(z)(1-u(z)) U_{s e}^{\prime}(u(z)] \exp \left(-\beta J_{s e} * u(z)+h(z)\right),\right. \text { and } \\
& G_{*}(z, t):=1-u(z, t)-u(z, t) \exp \left(-\beta J_{s f} * u(z)+h(z)\right) .
\end{aligned}
$$

In the case where only diffusion processes are included in (3.9) then $c=0$. Thus rescaling in time as $t \rightarrow t / N$ the $H_{*}(z)$ term becomes a first order correction,

$$
\partial_{t} u(z, t)+c_{0} \partial_{z} F_{*}(z, t)+c_{0} \frac{1}{N} \partial_{z} H_{*}(z, t)=0 .
$$

If adsorption/desorption processes are also included however the dominant term due to these dynamics is of order $O(1)$ and the full equation (3.9) is used without rescaling. In that case the diffusion process terms of order $1 / N$ and $1 / N^{2}$ become higher order corrections. We omit terms of order $1 / N^{2}$ in (3.9) and further examine the resulting PDE,

$$
\partial_{t} u(z, t)+c_{0} \frac{1}{N} \partial_{z} F_{*}(z, t)=c_{1} G_{*}(z, t) .
$$


3.2. Mesoscopic equations for ASEP with a look-ahead potential. In this section we outline the derivation of the equivalent mean field system under the special ASEP process introduced in section 2.2.1. In that respect, due to the onesided nature of the potential in (2.6) the spin-exchange process will only occur, using the notation from section 3.1 above, if $y>x$. Specifically, since $x$ and $y$ are nearest neighbors we must have $y=x+1$. The analysis of the derivation for this case is almost identical to that presented above in section 3.1 and is therefore left to the reader. We only outline the main results below. The main change is that equation (3.8) becomes

$$
\exp (-J \circ u):=\int_{z}^{\infty} J(y-z) u(y) d y .
$$

Therefore (3.6) simplifies to,

$$
\partial_{t} u(z, t)+c_{0} \frac{1}{N} \partial_{z} F_{o}(z, t)=c_{1} G_{o}(z, t)+O\left(1 / N^{2}\right)
$$

with $F_{o}$ and $G_{O}$ as follows:

$$
\begin{aligned}
& F_{o}(z, t):=u(z, t)(1-u(z, t)) \exp \left(-\beta\left(J_{s e} \circ u(z)+h(z)\right)\right), \text { and } \\
& G_{o}(z, t):=1-u(z, t)-u(z, t) \exp \left(-\beta\left(J_{s f} \circ u(z)+h(z)\right),\right.
\end{aligned}
$$

where we have employed the one sided convolution from (3.12) instead of (3.8). Note that once again we omit the $O\left(1 / N^{2}\right)$ term above and rescale time to absorb $1 / N$

$$
t \rightarrow N t
$$

in order to obtain the following partial differential equation,

$$
\partial_{t} u+c_{0} \partial_{z} F_{o}=c_{1} N G_{o}
$$

where $F_{o}$ and $G_{o}$ as in (3.13). Remark: see later results in section 4 where this partial differential operator (PDO) is approximated with various diffusive, dispersive equations.

3.3. Stochastic averaging. Another method which can often be used in obtaining closures for systems of stochastic differential equations involves the assumption of scale separation and ergodicity. We refer to [6] for these details. In particular we assume that the stochastic system evolves with a characteristic time which is much faster compared to that of the ODE. In this case we can employ a stochastic averaging principle utilizing the existing fast and slow scales [5]. The key ingredient for this method is the property of ergodicity for the stochastic process $\sigma$. We assume that the stochastic process $\sigma$ has a unique invariant measure which for the case of spin-flip/spin-exchange dynamics is described by $\mu_{\beta, N}(d \sigma)$ in (2.16). Assuming no phase transitions and fast relaxation time for the microscopic dynamics we can define

$$
\lim _{T \rightarrow \infty} \frac{1}{T} \int_{0}^{T} \mathcal{F}\left[Y, \bar{\sigma}_{t}\right] d s=\overline{\mathcal{F}}[Y]:=D \partial_{x}^{2} Y+A \partial_{x} Y+R Y+M u_{\beta, N}(h)+C
$$

where

$$
u_{\beta, N}(h)=E_{\mu_{\beta, N}} \bar{\sigma}_{t},
$$

and we assume that $N$ and $Y \in R^{2}$ is finite and fixed. Since $\mathcal{F}$ depends linearly on $u$ in $(2.2)$ we have

$$
E_{\mu_{\beta, N}} \mathcal{F}[Y, \bar{\sigma}]=\mathcal{F}\left[Y, u_{\beta, N}(h(Y))\right]
$$


Note that for spin-flip/spin-exchange interactions $u_{\beta, N}$ is analytic in $h$ for finite $N$. We therefore do not have to worry about the adverse effects of phase transitions often resulting from the $N \rightarrow \infty$ case. As a result the following reduced system is always well defined,

$$
\left\{\begin{array}{l}
\frac{d}{d t} \bar{y}_{t}=\frac{1}{\tau_{c}} \overline{\mathcal{F}}\left[\bar{y}_{t}\right] \\
\overline{\mathcal{F}}\left[\bar{y}_{t}\right]=D \partial_{x}^{2} \bar{y}_{t}+A \partial_{x} \bar{y}_{t}+R \bar{y}_{t}+M u_{\beta, N}\left(h\left(\bar{y}_{t}\right)\right)+C \quad \text { for } t \in[0, T], \\
\bar{y}_{0}=y
\end{array}\right.
$$

where $\bar{y}$ can be as close as we like to $Y$ : for any $\delta>0$,

$$
\lim _{\tau_{I} \rightarrow 0} P\left(\sup _{0 \leq t \leq T}|Y(t)-\bar{y}(t)|>\delta\right)=0,
$$

assuming an arbitrary bounded time interval $[0, T]$ and fixed number of interacting particles $N$ as well as characteristic time $\tau_{c}$. Phase transitions can still occur due to the coupling mechanism through the external potential $h$. These phase transitions however are attributed to hysteresis. We will provide examples where such phase transitions occur in section 5. For more details on phase transition phenomena under this case we also refer to work in [9]. Remark: we also note that it is not necessary to assume linearity in (3.15). By doing so however it becomes much easier to obtain analytical formulas which can later be used as benchmarks for comparisons. We also note that in the case of look-ahead potentials it is not obvious that the property of ergodicity is satisfied. For such potentials therefore stochastic averaging closures may not be applicable.

\section{Linearized stability analysis of the mesoscopic models}

In order to better understand the overall behavior for system (2.1) we produce PDEs which approximate the mesoscopic equations obtained previously in section 3 . To that endeavor we employ linearization techniques as well as expansions around constant states. In section 3 above we obtained the following nonlinear, time rescaled $(t \rightarrow N t)$, integrodifferential approximation in the case of symmetric interaction potentials, with contributions from both spin-flip and spin-exchange dynamics:

$$
\partial_{t} u(z, t)+c_{0} \partial_{z} F_{*}(z, t)=c_{1} N G_{*}(z, t) .
$$

Here $u(z, t) \approx E \sigma_{t}(z)$ denotes the average density and

$$
\begin{aligned}
& F_{*}(z, t)=u(z, t)(1-u(z, t)) \exp \left(-\beta\left(J_{s e} * u(z)+h(z)\right)\right) \\
& G_{*}(z, t)=1-u(z, t)-u(z, t) \exp \left(-\beta\left(J_{s f} * u(z)+h(z)\right),\right.
\end{aligned}
$$

where for $J \equiv J_{s e}$ or $J \equiv J_{s f}$ we have

$$
\beta J * u(z)=\beta \int_{-\infty}^{\infty} J(y-z) u(y) d y .
$$

We will now carry out expansions of the PDE/integrodifferential (4.1) in order to obtain through linearizations subsequent PDE approximations. Note that if more detail is required one can also carry out the same analysis for (4.1) without the partial differential operator (PDO) approximation (4.3) below. We further note that this analysis can be relevant for either attractive or repulsive potentials [19]. 
4.1. Differences between symmetric and look-ahead potentials. The main difference between look-ahead and symmetric potentials is attributed to the convolution mechanism (4.2). This mechanism is responsible for accounting for all local contributions and differentiating between symmetric and look-ahead interactions as follows:

- In the case of symmetric potentials, we expand (4.2),

$$
\begin{aligned}
\beta J * v(z) & :=\int_{-\infty}^{\infty} \beta J(y-z) u(y) d y={ }^{x=y-z} \\
& =\int_{-\infty}^{\infty} \beta J(x) u(x+z) d x=\bar{J}_{0} u+\bar{J}_{1} u_{x}+\bar{J}_{2} u_{x x}+\bar{J}_{3} u_{x x x}+\ldots,
\end{aligned}
$$

where for $J>0$ gives (i.e., assuming that $J \equiv J_{s e}$ and $J \equiv J_{s f}$ are positive),

$$
\begin{aligned}
& \bar{J}_{0}=\beta \int_{-\infty}^{\infty} J(x) d x>0, \quad \bar{J}_{1} \quad=\beta \int_{-\infty}^{\infty} x J(x) d x=0 \\
& \bar{J}_{2}=\beta \int_{-\infty}^{\infty} \frac{x^{2}}{2} J(x) d x>0, \quad \bar{J}_{3}=\beta \int_{-\infty}^{\infty} \frac{x^{3}}{6} J(x) d x=0 .
\end{aligned}
$$

- In the case of look-ahead potentials, (4.2) becomes

$$
\begin{aligned}
\beta J \circ v(z): & =\int_{0}^{\infty} \beta J(y-z) u(y) d y={ }^{x=y-z} \\
& =\int_{0}^{\infty} \beta J(x) u(x+z) d x=\hat{J}_{0} u+\hat{J}_{1} u_{x}+\hat{J}_{2} u_{x x}+\hat{J}_{3} u_{x x x}+\ldots,
\end{aligned}
$$

where

$$
\begin{aligned}
& \hat{J}_{0}=\beta \int_{0}^{\infty} J(x) d x>0, \quad \hat{J}_{1}=\beta \int_{0}^{\infty} x J(x) d x>0 \\
& \hat{J}_{2}=\beta \int_{0}^{\infty} \frac{x^{2}}{2} J(x) d x>0, \quad \hat{J}_{3}=\beta \int_{0}^{\infty} \frac{x^{3}}{6} J(x) d x>0
\end{aligned}
$$

Note that the correspondence between symmetric (4.4) and look-ahead parameters (4.6) in the expansion of the interaction potentials above provides the following relationship,

$$
\hat{J}_{0}=\frac{\bar{J}_{0}}{2}, \hat{J}_{2}=\frac{\bar{J}_{2}}{2}, \text { etc. }
$$

We also note that although for symmetric potentials, $\bar{J}_{1}=\bar{J}_{3}=\cdots=0$, this relationship is not necessarily true for look-ahead potentials, $\hat{J}_{1}, \hat{J}_{3}, \ldots$. We apply either (4.3) or (4.5) and approximate the exponential from

$$
\begin{aligned}
\exp (-\beta(J * u+h)) & \approx e^{-J_{0} u-\beta h} e^{-J_{1} u_{z}-J_{2} u_{z z}-J_{3} u_{z z z}} \\
& \approx e^{-J_{0} u-\beta h}\left[1-J_{1} u_{z}-J_{2} u_{z z}-J_{3} u_{z z z}\right],
\end{aligned}
$$

where $J_{n} \equiv \bar{J}_{n}$ or $J_{n} \equiv \hat{J}_{n}$ for $n=0,1,2, \ldots$ depending on whether symmetric or lookahead potentials are used. As a result we obtain the following approximation for (4.1),

$$
\partial_{t} u+F^{a}=F^{r}+F^{d}+F^{s},
$$

where we define (and highlight the contributions of each process)

spin-exchange spin-flip.

$$
\begin{array}{lr}
F^{r}:= & c_{1} N\left(1-u-u e^{-J_{0} u} e^{-\beta h}\right) \\
F^{a}:=c_{0}\left(u(1-u) e^{-J_{0} u} e^{-\beta h}\right)_{z} & -c_{1} N u J_{1} e^{-J_{0} v} e^{-\beta h} v_{z} \\
F^{d}:=c_{0}\left(u(1-u) J_{1} u_{z} e^{-J_{0} u} e^{-\beta h}\right)_{z}+c_{1} N u J_{2} e^{J_{0} u} e^{-\beta h} u_{z z} \\
F^{s}:=c_{0}\left(u(1-u) J_{2} u_{z z} e^{-J_{0} u} e^{-\beta h}\right)_{z}+c_{1} N u J_{3} e^{J_{0} u} e^{-\beta h} u_{z z z},
\end{array}
$$


The PDE can be written all together as follows,

$$
\begin{aligned}
\frac{d}{d t} u(z, t) & +\overbrace{c_{0}\left[u(1-u) e^{-J_{0} u} e^{-\beta h}\right]_{z}-c_{1} N J_{1} e^{-J_{0} u} e^{-\beta h} u u_{z}}^{\text {Advection }}= \\
& =\begin{array}{c}
c_{1} N\left(1-u-u e^{-J_{0} u} e^{-\beta h}\right) \\
\text { Reaction }
\end{array} \\
& +\overbrace{c_{0}\left(u(1-u) J_{1} u_{z} e^{-J_{0} u} e^{-\beta h}\right)_{z}+c_{1} N J_{2} e^{-J_{0} u} e^{-\beta h} u u_{z z}}^{\text {Dispersion }} \\
& +\overbrace{c_{0}\left(u(1-u) J_{2} u_{z z} e^{-J_{0} u} e^{-\beta h}\right)_{z}+c_{1} N u J_{3} e^{-J_{0} u} e^{-\beta h} u_{z z z}}^{\text {Difion }}
\end{aligned}
$$

Note that the corresponding integrodifferential equations based on each of the two stochastic mechanisms have the following form:

For, non-conservative, spin-flip dynamics only (i.e., $c_{0}=0$ ):

$$
\begin{aligned}
& \frac{d}{d t} u(z, t)-c_{1} N J_{1} e^{-J_{0} u} e^{-\beta h} u u_{z} \\
= & c_{1} N\left(1-u-u e^{-J_{0} u} e^{-\beta h}\right)+c_{1} N J_{2} e^{-J_{0} u} e^{-\beta h} u u_{z z}+c_{1} N J_{3} e^{-J_{0} u} e^{-\beta h} u u_{z z z} .
\end{aligned}
$$

For, conservative, spin-exchange dynamics only (i.e., $c_{1}=0$ ):

$$
\begin{aligned}
& \frac{d}{d t} u(z, t)+c_{0}\left[u(1-u) e^{-J_{0} u} e^{-\beta h}\right]_{z} \\
= & c_{0}\left(u(1-u) J_{1} u_{z} e^{-J_{0} u} e^{-\beta h}\right)_{z}+c_{0}\left(u(1-u) J_{2} u_{z z} e^{-J_{0} u} e^{-\beta h}\right)_{z} .
\end{aligned}
$$

Below, we carry out stability analysis for parts of (4.10), with either (4.11) or (4.12) in an effort to better understand the behavior of each stochastic mechanism. The purpose of this analysis is to identify critical parameter regimes for which small perturbations can produce substantial departures from the expected mean field behavior.

4.2. Constant States. Typical solution behavior for system (2.2) can be understood by examining its constant states. We provide a brief outline of such states here. Details for solution behavior of hybrid systems involving ODEs coupled to stochastic noise models can be found in [6]. For similar systems undergoing phase transitions details can be found in [8]. We first note that the stochastic system already described in section 2 may undergo a phase transition if $\beta J_{0} \geq \beta_{c} J_{0}:=4$, where $\beta_{c}$ denotes a critical temperature threshold. In that respect the constant states of (2.2), which includes effects due to a possible phase transition, are described by

$$
\begin{aligned}
\frac{d}{d t} Y & =R Y+M u+C \\
\frac{d}{d t} u & =g(u)-Y,
\end{aligned}
$$

where $g(u)$ is a cubic polynomial in $u$. Note that for $g(u)=u(a-u)(u-1)(4.13)$ is equivalent to the FitzHugh Nagumo (FHN) equation [19]. The constant states of (4.13) are changing depending on the value of $\beta J_{0}$. If $\beta J_{0}<4$ then the bottom equation of (2.2) becomes monotone and has a single stable, excitable state as can be seen in figure 4.1a. If on the other hand we are in the phase transition regime $\beta J_{0} \geq 4$ then the stability profile may have multiple states exhibiting excitable, oscillatory 

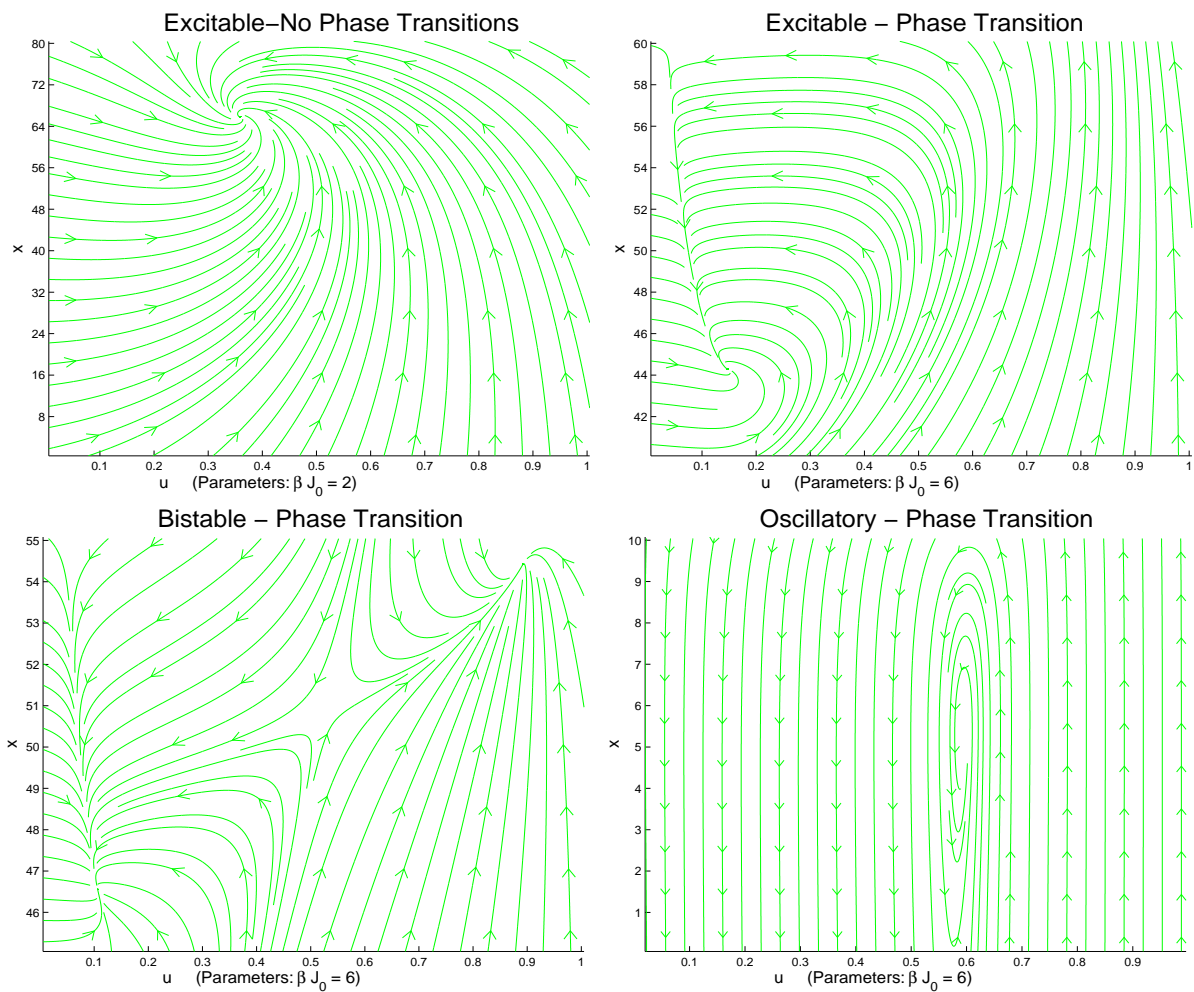

FIG. 4.1. Direction field and phase portrait for mean field system (4.13) under different parameter regimes. Cases of excitability for no phase transitions, $\beta \bar{J}_{0}=2$, and excitability, bistability and oscillations respectively for phase transition regime with $\beta \bar{J}_{0}=6$. Compare with pp. 328-329 of [19]. The behavior shown here is exactly the same for the FitzHugh-Nagumo equation shown in (4.13).

or bistable behavior depending on the parameters used. See figure $4.1 \mathrm{~b}, \mathrm{c}$, d for those examples. In the ensuing stability analysis we carry out expansions around the constant states of (2.2). The results will be shown to carry through and agree with the behavior of the solutions for (4.13). In the following presentation we denote those constant states as $u_{0}, Y_{0}$.

4.3. Linear stability for system with symmetric dynamics. We now examine the stability of the coupled system which includes some of the major mechanisms, advection, diffusion, and dispersion, contributed from the stochastic piece in (4.10). In this section we examine stability under the case of symmetric potentials $J$ as in (4.2). We assume for simplicity that the coupling is imposed only on the spin-flip and not the spin-exchange dynamics. The analysis however can be carried out in exactly the same way in the case that $h(Y)$ is included in both potentials. The expansion for the corresponding potentials was provided in (4.3) and (4.8). Thus, 
using these contributions from (4.10) in our coupled system we obtain

$$
\left\{\begin{aligned}
\partial_{t} Y= & D \partial_{x x} Y+A \partial_{x} Y+R Y+M u+C \\
\partial_{t} u= & d_{0} \partial_{x}\left[u(1-u) e^{-\bar{J}_{0} u}\right]+d_{1} \partial_{x}\left[u(1-u) e^{-\bar{J}_{0} u} \partial_{x} u\right] \\
& +d_{2} \partial_{x}\left[u(1-u) e^{-\bar{J}_{0} u} \partial_{x x} u\right]+d_{3}\left(1-u-u \exp \left[-\bar{J}_{0} u+\beta h(Y)\right]\right),
\end{aligned}\right.
$$

where $d_{0}, d_{1}, d_{2}$, and $d_{3}$ are chosen appropriately from (4.10) and depend on $c_{0}, c_{1}$, and $N$. Other free parameters will be chosen in the numerical section later. Here $h(Y)=$ $\alpha(Y-\gamma)$ and the parameters $\alpha$ and $\gamma$ are to be specified in the numerical section and depend on the type of behavior we wish the system to exhibit. Note that contributions of $\bar{J}_{1}, \bar{J}_{3}, \ldots$ are zero due to the symmetry of the potential in (4.3). We consider all non-zero terms greater than or equal to $\bar{J}_{2}$ as higher order corrections to (4.14) and therefore we do not present them here. Also note that all coupling contributions from the solution $Y$ of the PDE to the stochastic piece are accounted for in a single term originating from the spin-flip dynamics. We assume no coupling contributions from the spin-exchange Arrhenius dynamics. We apply a small perturbation around the known equilibrium solutions $u_{0}, Y_{0}$,

$$
\begin{aligned}
& Y=Y_{0}+Y_{1} e^{s t+I k x} \\
& u=u_{0}+u_{1} e^{s t+I k x}
\end{aligned}
$$

Note that it is sufficient for now to apply the same perturbation in both $u$ and $Y$. We obtain a simplified Jacobian of the form

$$
\overline{\mathcal{J}}=\left[\begin{array}{ll}
\bar{F}_{Y_{1}} & \bar{F}_{u_{1}} \\
\bar{G}_{Y_{1}} & \bar{G}_{u_{1}}
\end{array}\right]:=\left[\begin{array}{ll}
s+D k^{2}-I A k-R & -M \\
\overline{\mathcal{E}}\left(\bar{J}_{0}\right) \alpha k I u_{0}\left[u_{0}-1\right] s-\overline{\mathcal{E}}\left(\bar{J}_{0}\right) I k\left[1-\left(\bar{J}_{0}+2\right) u_{0}+\bar{J}_{0} u_{0}^{2}\right]
\end{array}\right],
$$

where $\overline{\mathcal{E}}\left(\bar{J}_{0}\right) \equiv \overline{\mathcal{E}}_{u_{0}, Y_{0}}\left(\bar{J}_{0}\right):=\exp \left(-\bar{J}_{0} u_{0}+\beta h\left(Y_{0}\right)\right)$. Note that we consider terms greater than or equal to $\bar{J}_{2}$ as correction terms for this approximation. A specific example of this Jacobian is provided below for $\bar{J}_{0}=1$. To simplify the presentation we apply values for some variables which exhibit excitable behavior. In this case the Jacobian has the form

$$
\overline{\mathcal{J}}=\left[\begin{array}{cc}
s+D k^{2}-I A k-R & -M \\
.05 & s-.52 I k d_{0}+.1 d_{1} k^{2}+.1 d_{2} I k^{3}+1.9
\end{array}\right] .
$$

We also omitted terms $J_{2}$ and higher for convenience although they can become important, as correction terms, in higher order approximations. Based on our analysis from this system we find that the effects of advection and dispersion are in general counteracting each other. Furthermore, although not surprising, we also find that diffusion due to its natural mixing improves the stability of an already stable solution. We also note that although the analysis carried out above is general it is presented here for the case of a classical type interaction potential as given in (2.4). The case of the new, one-sided, potential (2.6) is analyzed below:

4.4. Linear stability for system with look-ahead advection. We now examine the stability for system (2.2) as approximated through (4.10) for the case of the one-sided look-ahead interaction potential (2.6) and (3.12). The only difference from the derivation leading to equation (4.10) is that we now use the one-sided ASEP process which in effect alters the description of the convolution in (4.2) in the way presented in (4.6). Again, for simplicity, we assume that the coupling $h(Y)$ is 
only through the spin-flip and not the spin-exchange dynamics. The corresponding expansions can be found in (4.5) and (4.8). The equivalent mean field version for a system with look-ahead potentials including contributions up to and including $\hat{J}_{1}$ is given below,

$$
\left\{\begin{aligned}
\partial_{t} Y= & D \partial_{x x} Y+A \partial_{x} Y+R Y+M u+C \\
\partial_{t} u= & d_{0} \partial_{x}\left[u(1-u) e^{-\left(\hat{J}_{0}+\hat{J}_{1}\right) u}\right]+d_{1} \partial_{x}\left[u(1-u) e^{-\left(\hat{J}_{0}+\hat{J}_{1}\right) u} \partial_{x} u\right] \\
& +d_{2} \partial_{x}\left[u(1-u) e^{-\left(\hat{J}_{0}+\hat{J}_{1}\right) u} \partial_{x x} u\right]+d_{3}\left(1-u-u \exp \left[-\left(\hat{J}_{0}+\hat{J}_{1}\right) u+\beta h(Y)\right]\right)
\end{aligned}\right.
$$

where, once again, the parameters $d_{0}, d_{1}, d_{2}$, and $d_{3}$ depend explicitly on $c_{0}, c_{1}$, and $N$ from (4.10). The Jacobian for this system is

$$
\begin{aligned}
\hat{\mathcal{J}} & =\left[\begin{array}{ll}
\hat{F}_{Y_{1}} & \hat{F}_{u_{1}} \\
\hat{G}_{Y_{1}} & \hat{G}_{u_{1}}
\end{array}\right] \\
& :=\left[\begin{array}{cc}
s+D k^{2}-I A k-R & -M \\
\hat{\mathcal{E}}\left(\hat{J}_{0}, \hat{J}_{1}\right) \alpha k I u_{0}\left[u_{0}-1\right] s-\hat{\mathcal{E}}\left(\hat{J}_{0}, \hat{J}_{1}\right) I k\left[1-\left(\hat{J}_{0}+\hat{J}_{1}+2\right) u_{0}+\left(\hat{J}_{0}+\hat{J}_{1}\right) u_{0}^{2}\right]
\end{array}\right]
\end{aligned}
$$

where $\hat{\mathcal{E}}\left(\hat{J}_{0}, \hat{J}_{1}\right) \equiv \hat{\mathcal{E}}_{u_{0}, Y_{0}}\left(\hat{J}_{0}, \hat{J}_{1}\right):=\exp \left(-\left(\hat{J}_{0}+\hat{J}_{1}\right) u_{0}+\beta h\left(Y_{0}\right)\right)$. Terms greater than or equal to $\hat{J}_{2}$ are considered to be correction terms here. The eigenvalues of of this Jacobian are influenced by the, non-zero, perturbation $\hat{J}_{1}$ due to the look-ahead dynamics. Notice that such a perturbation is impossible for symmetric potentials since $\hat{J}_{1}=0$. As an example of (4.17) we display below, in figure 4.2, the real part of the first and second eigenvalues corresponding to excitable type of behavior, for appropriate values of the constant parameters.
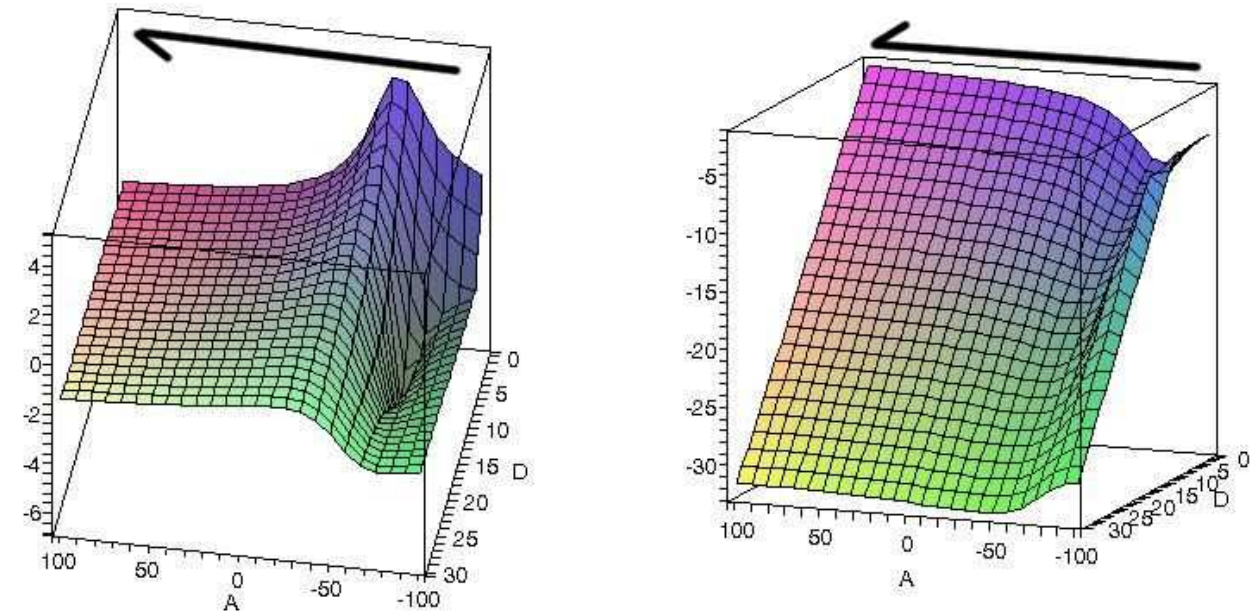

FIG. 4.2. Linear stability behavior of the Jacobian (4.17) for look-ahead dynamics. We display the real part of the first (left) and second (right) eigenvalues with respect to advection $A$ and diffusion $D$. The arrow displays a lateral move of the surface as $d_{0}$, in (4.16), ranges from-10 to 10 . 


\section{Stochastic effects}

In this section we use Monte Carlo methods to simulate the full system (2.2) for those critical parameter regimes identified in section 4 . Thus, as linearized stability analysis points out, closures of the original system (2.2), although useful, must be applied with care especially if the system is undergoing transient or long-time dynamics. Furthermore, look-ahead dynamics are responsible for dramatically changing the behavior of the solutions for the coupled system. A number of new states emerge as a result of applying the look-ahead dynamics which evolve through translations, synchronization, intermittency, focusing/defocusing effects, and more. Those states are not part of the usual mean field profile for our system but rather a result of the one sided look-ahead potential of the stochastic component of the coupled system. We present below several examples which display vast differences in the behavior of the solutions for our system due to influences originating in the stochastic component and in particular in the look-ahead mechanism. The simulations which are presented below focus in the following topics:

- differences between solutions of the PDE versus the ODE

- effects attributed to noise. This includes differences between mean field and stochastic solutions

- effects due to lateral lattice interactions.

5.1. Instability attributed to advection. In this section we examine the influence of advection $A$ and stochasticity on the stability of the solutions. We discover that large advection, acting as a strong stirring mechanism, becomes a catalyst to promote stability in the solutions. In contrast, low advection in conjunction with stochastic perturbations can produce large excitations from equilibrium and eventual instability. Specifically we display two examples in figure 5.1 where we have fixed all parameters exactly the same except for the advection parameter $A$. On the top row of figure 5.1 we display the mean field (left column) and stochastic (right column) solutions of our system for a large advective parameter, $A=20$, while the exact same results are displayed on the bottom row for smaller advection value of $A=.2$. Clearly the stochastic solution in the bottom part of that figure, corresponding to the small advection value $A=.2$, is unstable. Overall we would therefore describe the behavior of this system as, a stochastically induced, convective instability phenomenon. This instability is attributed both to convection as well as stochastic perturbations. We should also point out the the equivalent system for $A=0$, not presented here, produced stable solutions as well. Note that the instability we observe here is not that of a blowup type but instead one which does not allow the system to relax to its constant state. For values of $A \leq \sigma / k$ we observe that the system is constantly pushed away from its equilibrium. This behavior is what we call convective instability.

5.2. Random switching between stochastic waves.

In this example we present a phase transition phenomenon which is induced by stochastic nonlinear effects. Specifically the system, due to strong particle interactions and lookahead dynamics, is undergoing a phase change, or transition, from one orbit to another as can be seen in the phase plot of figure 5.2. Note that the system reorganizes in time and attains a new traveling wave solution which reaches a pointedly different wave speed than the solution before it had. We also note that in order for such a phase transition to occur the system must undergo a reorganization and synchronization in space and time in order to achieve the new state. 

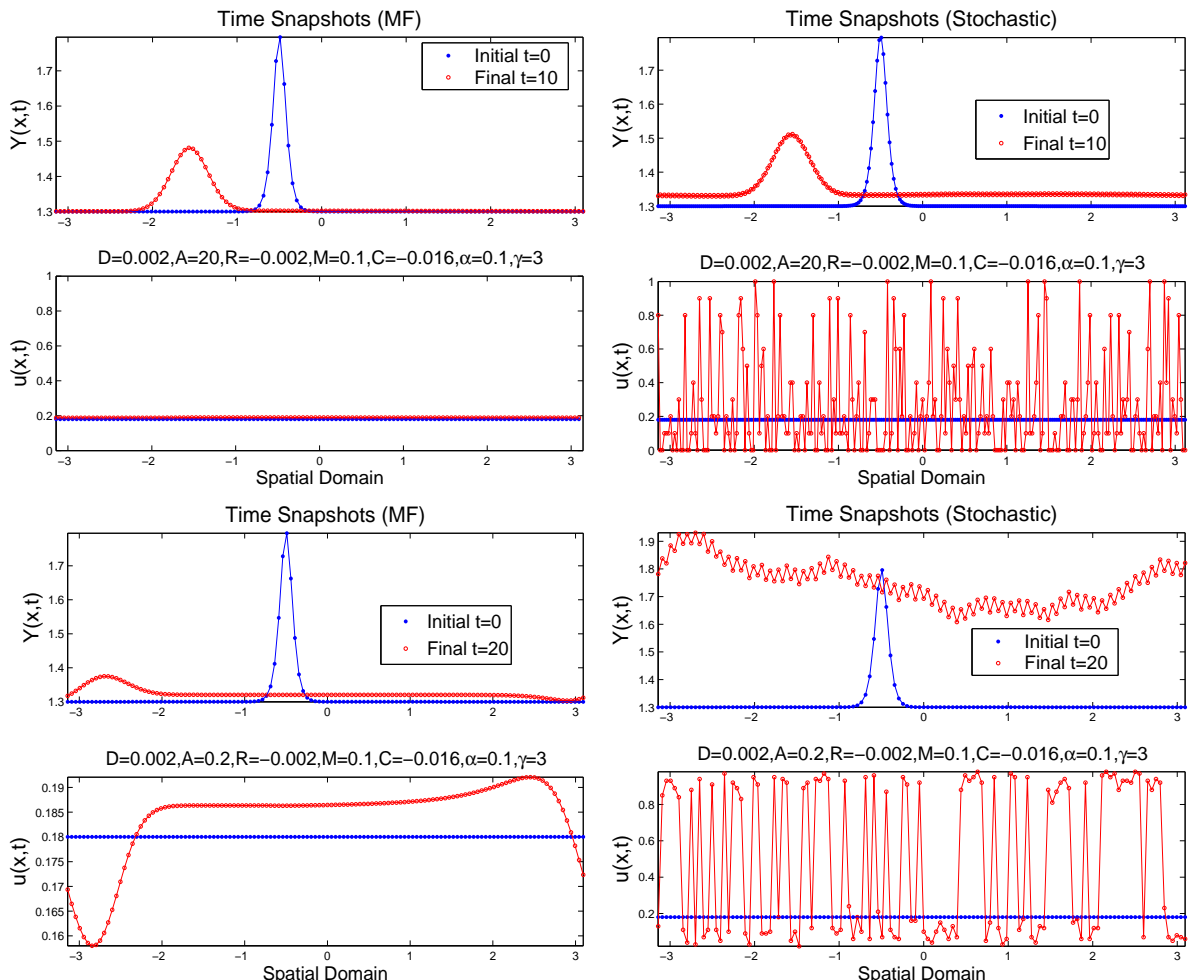

FIG. 5.1. Instability due to convection. Solution profiles are displayed for both, initial and final times, for the excitable regime. Top row displays results for high advection speed $A=20$. Bottom row for lower advection speed $A=.2$. Mean-field system (4.14) on the left and full stochastic coarse grained system (2.2) on the right. Convective instability is displayed in subfigure (d) which corresponds to the case of low advection value $A=.2$. The case of $A=20$ produced stable solutions.

In a relevant example, in figure 5.3, we observe the influence of look-ahead dynamics responsible for generating a traveling wave on the stochastic component of the coupled system which, in turn, produces an overall traveling wave solution for the system. In contrast, the corresponding solution for the system without look-ahead, also included in figure 5.3, displays non-moving constant structures.

5.3. Altering the solution profile. We present further numerical evidence of the role of look-ahead dynamics in altering the original system profile. Specifically, in figure 5.4 we observe how look-ahead dynamics perturb the system solution just enough to alter its profile from excitable to oscillatory. The stability analysis which is also included in figure 4.2 sheds further insight as to what kind of behavior we should expect based on the values of advection $A$ and diffusion $D$. Based on this analysis it is clear that the overall behavior of the PDE depends on the real parts of each of the two eigenvalues for given values of $A$ and $D$ in relation to noise levels of the stochastic piece. With enough noise and assuming that the real parts are not too negative then the imaginary parts of the eigenvalues can take over and produce the oscillation observed in some of our simulations. Our findings therefore suggest:

excitability $\rightarrow$ spikes in $u \rightarrow$ spatial structures in $Y$ (highly oscillatory). 

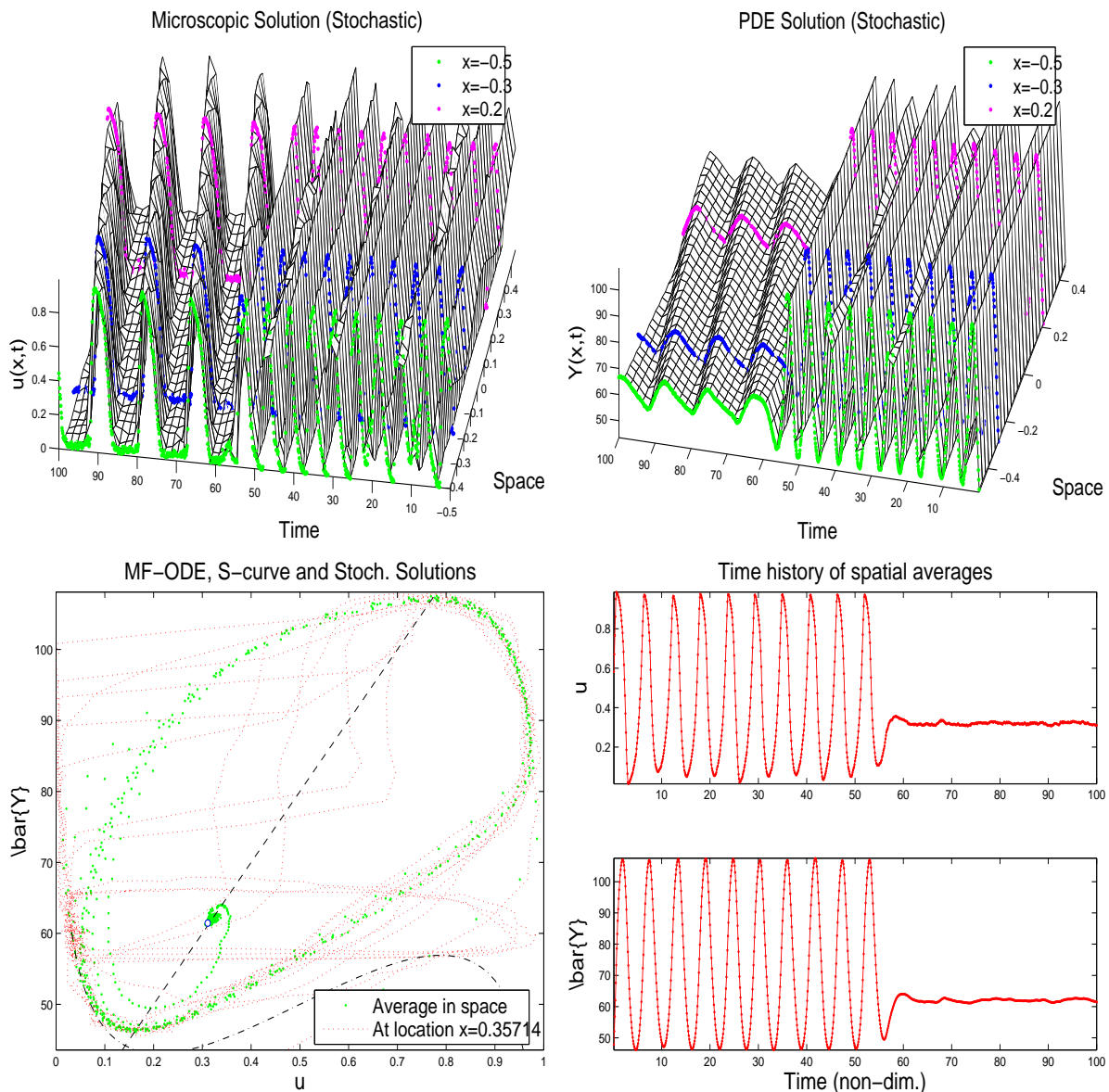

FIG. 5.2. Random switching resulting in attaining different orbits. Solutions presented for case of full hybrid system (2.2) with look-ahead dynamics. On left bottom plot the dotted line indicates the mean field ODE profile corresponding to system (4.13).

Note that spikes in $u$, induced by stochastic noise, will not develop into "island" type structures as in the nearest neighbor case presented later in figure 5.7.

5.4. Random pulses. The stochastic coupled system is responsible for rich solution behavior including random pulses. Such an effect is presented in the solution of system (2.2) in the right subplot of figure 5.5. In that figure we observe synchronization in both space and time which is responsible for the eventual reorganization of the solution into a pulse. The look-ahead potential has contributed substantially towards the development of such random single pulses. Without these one-sided dynamics the pulse does not occur. This was observed in several examples one of which is shown in the left part of figure 5.5. The only difference between this and the right part of figure 5.5 is the inclusion of the look-ahead potential.

5.5. Profile translations. In the next numerical example we study a complete shift in the phase plane solutions attributed, as we might expect by now, to the look-ahead dynamics inclusion in the stochastic component. In figure 5.6 the phase 

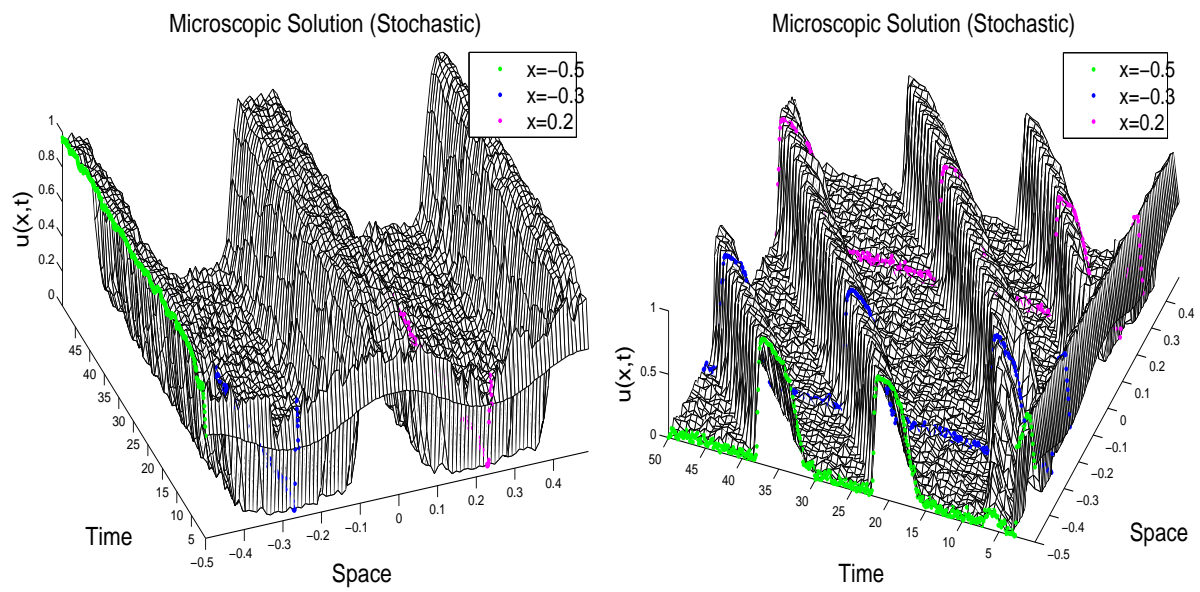

FIG. 5.3. Solution u from (2.1). Look-ahead dynamics, on the right, result in traveling waves in the stochastic component. Left: no look-ahead. All other parameters are kept the same in both figures.
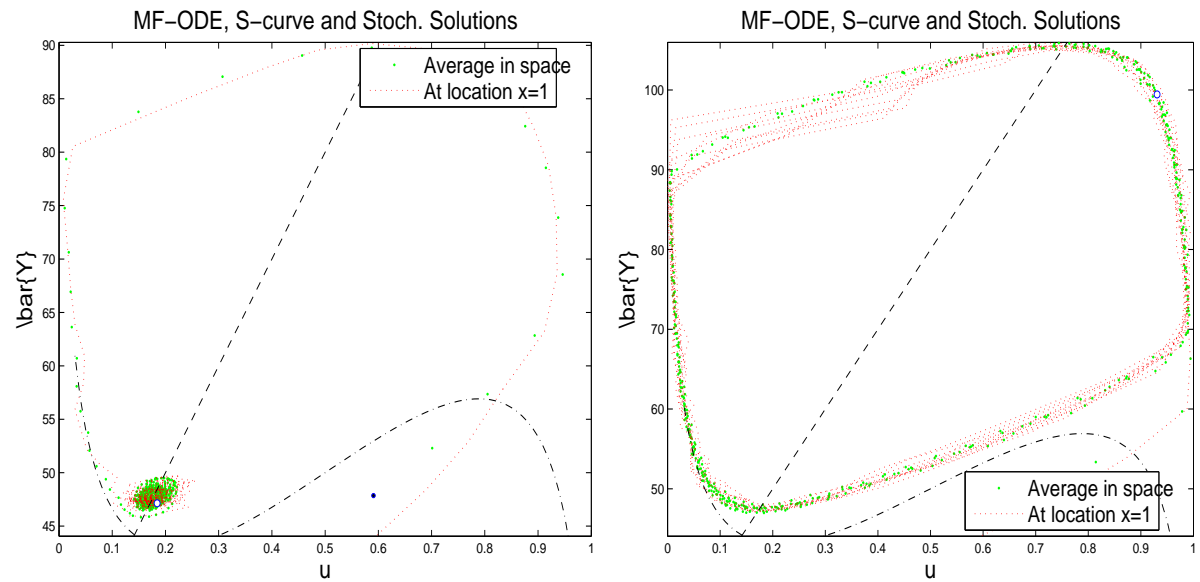

FIG. 5.4. Phase plane. Look-ahead dynamics, on the right, alter the solution profile from excitable to oscillatory. Left: usual potential. The only difference in creating the two figures above is that on the left no look-ahead is used while on the right we include look-ahead dynamics. The mean field ODE profile corresponding to (4.13) is also noted with a doted line in the figures above.

plane reveals that the original oscillatory solution has shifted due to the look-ahead potential. Overall, we observe that, using the look-ahead mechanism we can completely alter the system dynamics. The system solution therefore can be perturbed to eventually produce bistable, oscillatory, pulsating and excitable type behavior even though this was not even possible for the original system profile according to either mean field theory or stability analysis. From our simulations we observe that this can be achieved by changing either the look-ahead interaction range and/or the relaxation time $\tau_{I}$ of the stochastic component. 

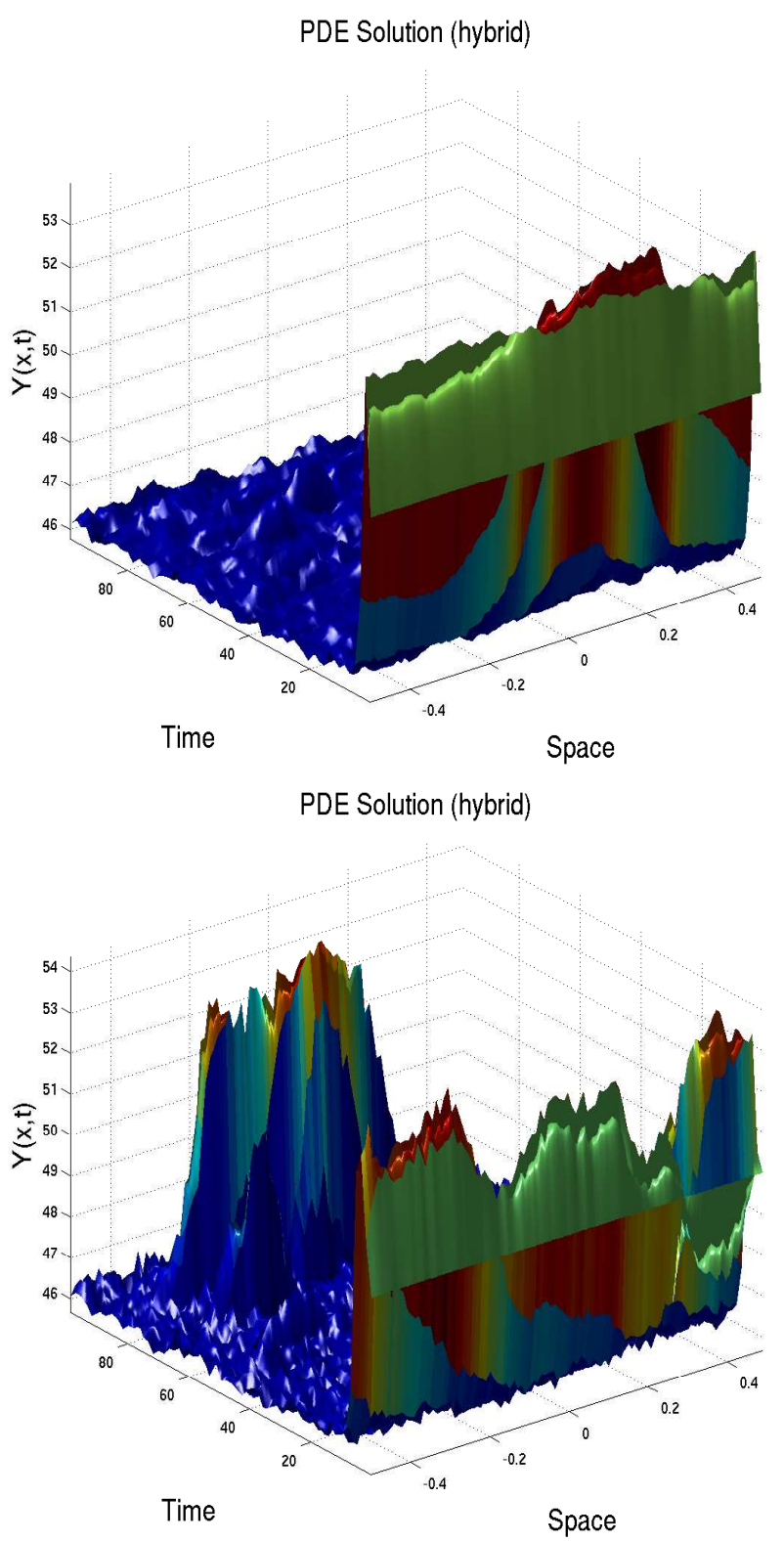

FIG. 5.5. PDE solution. Time, space synchronization which evolves into a single pulse attributed to use of the one-sided look-ahead dynamics. The only difference in creating the two figures above is that on the left no look-ahead is used while on the right we include look-ahead dynamics.

5.6. Generation of island like structures. In this section we examine the behavior of our system under the influence of spatial interactions due to inclusion of nearest neighbor interactions in space. This is in constrast to birth-death type dynamics which do not involve information from neighbors. We present results in figure 5.7 for the excitable regime and compare the solutions against the equivalent solutions 

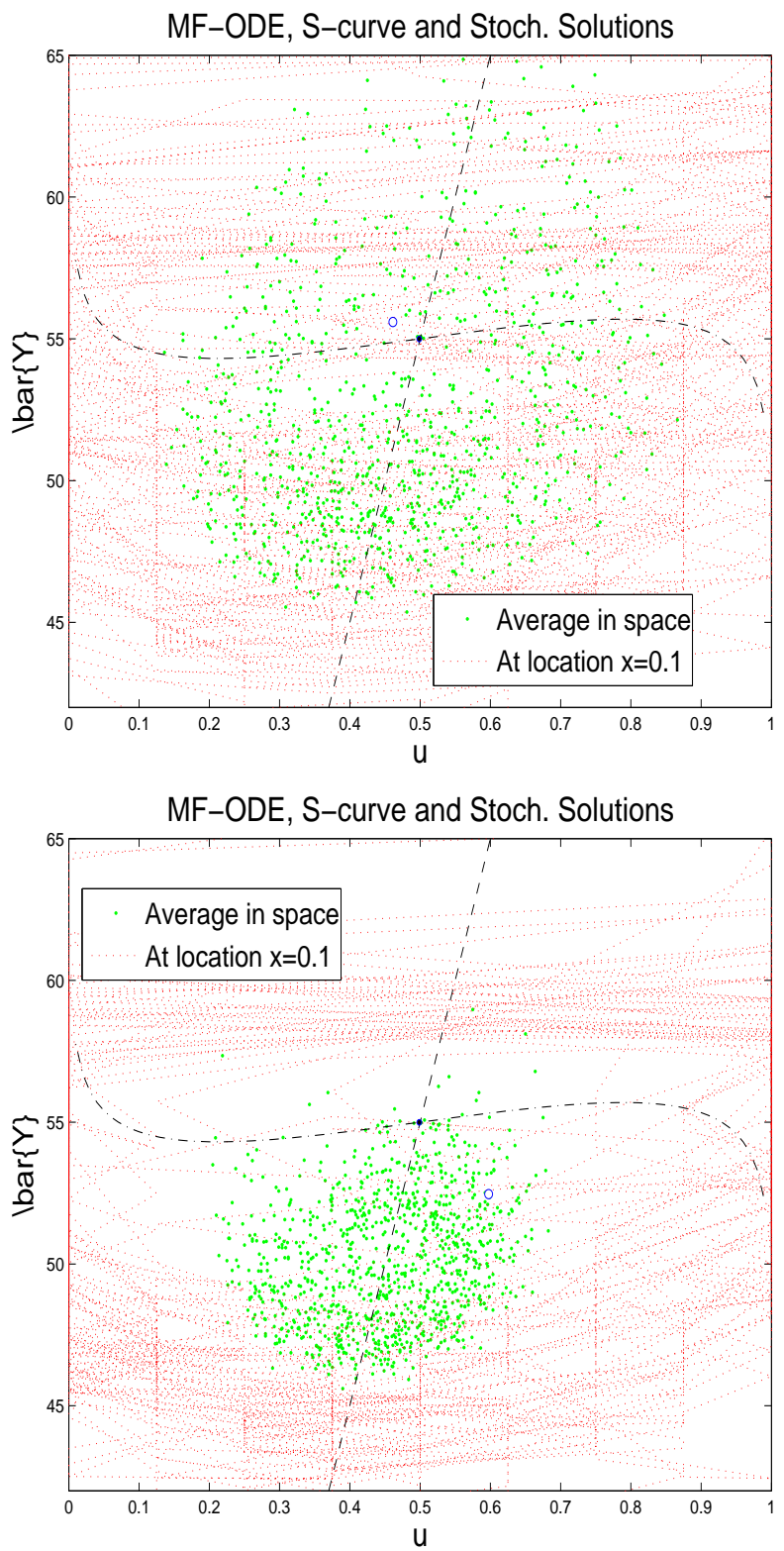

FIG. 5.6. Oscillatory regime. Phase plane solutions display a shift downwards attributed to inclusion of the look-ahead potential. The only difference in creating the two figures above is that on the left no look-ahead is used while on the right we include look-ahead dynamics. The mean field $O D E$ profile is also indicated in the plots above with a dotted line.

in the case of birth death dynamics (no lateral interactions in space). We further note that in this example we implement a symmetric interaction potential (2.4). Note that the behavior due to the spatial nearest neighbor mechanism resulted in creating sustainable 'island' type structures in space and time for $u$, as well as eliminating the previously observed oscillations in space in $Y$ (not shown here). Therefore in the 
excitable regime we find that,

nearest neighbor interactions $\rightarrow$ 'island' structures $\rightarrow$ smoother mean field behavior destroy noisy spikes in $u \quad$ are created in $u \quad$ in $Y$ (no oscillations)

See figure 5.7 for comparisons between birth-death and nearest neighbor interactions in the excitable regime. Overall we find that when comparing, under similar conditions, birth-death vs nearest neighbor dynamics the former are observed to be much more susceptible to noise. In general we observe that nearest neighbor interactions allow synchronization and spatial reorganization to occur in the solution while attaining its equilibrium.
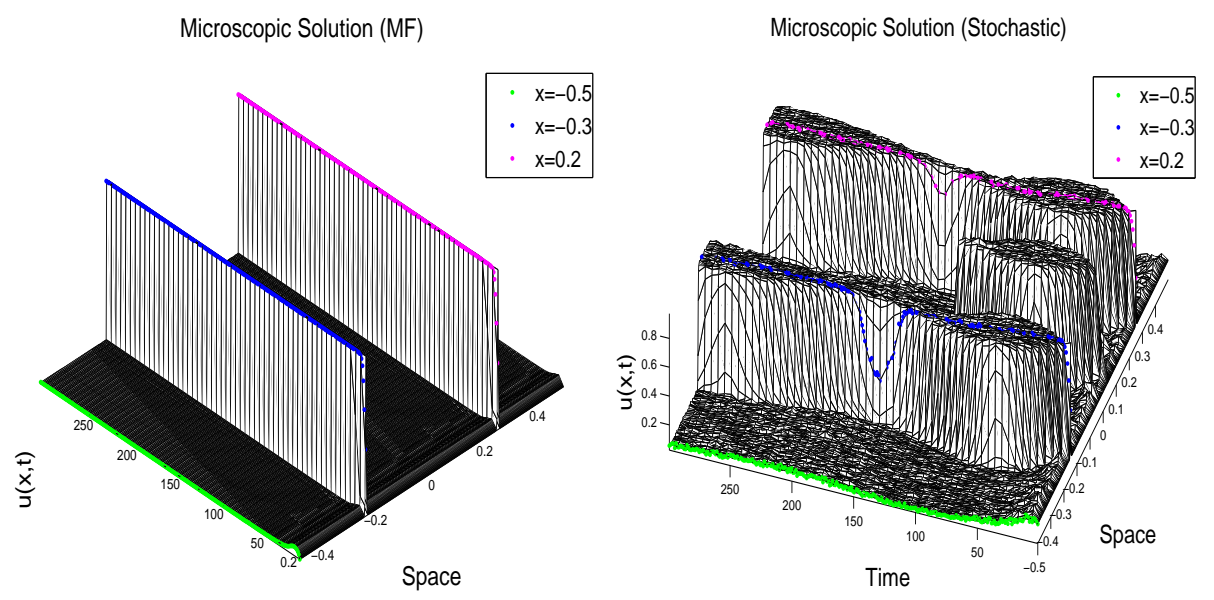

FIG. 5.7. Lateral interactions resulting from nearest neighbor interactions. Left: solution u from mean field system (4.14). Right: solution of system (2.2) depicting evolution of island type structures due to nearest neighbor interactions.

\section{General comments and remarks}

We studied a hybrid system consisting of a PDE and a stochastic noise model. The stochastic model is coupled directly to the PDE through a linear term while the PDE is coupled to the stochastic through its local interaction potential. Asymptotic derivations were carried out in order to produce the mesoscopic equivalent PDEs corresponding to our hybrid system. The hybrid system as well as the mean field closures were considered under extreme cases undergoing random wave switching and metastability. Mean field closures can not be expected to be able to capture the solution of such systems and in fact fail. In contrast we presented a stochastic type hierarchical closure which is able to produce approximate solutions under all parameter regimes. We examined the overall system behavior under a variety of stochastic dynamics including spin-flip and spin-exchange interactions. In the context of this hybrid system we also presented a new type of look-ahead interactions which produce advective effects in the stochastic component. The overall behavior of the system changed dramatically because of the inclusion of such dynamics. Through linear stability analysis we were able to observe that unexpected solution profiles emerged. Numerical simulations further revealed rich solution behavior comprised of random pulses, figure 5.5, and random switching between orbits, figure 5.2. Some further findings and remarks are included below: 
- Mean field closures cannot be trusted to produce system solutions even if the system is considered under strong flows induced from either the PDE or the stochastic. Fluctuations and their nonlinear effects are still important.

- When nearest neighbor interactions are considered nucleation phenomena occur and creation of island type structures similar to those presented in figure 5.7 are possible. Deterministic closures can not capture these random structures. An interesting follow up question would be to understand for what strength of interactions $J$ does the nucleation phenomenon are subsequent generation of these structures occur.

Acknowledgments. The research of M.A.K. is partially supported by NSFDMS-0413864, NSF-DMS-0715125 and DOE DE-FG02-05ER25702. The research of A.J.M. is partially supported by ONR N00014-96-1-0043, NSF-DMS-9972865 and NSF-CMG-0222133. The research of A.S is partially supported by NSF-DMS $0606807 / 0836699$ and by funds provided by the University of North Carolina at Charlotte

\section{REFERENCES}

[1] T. Alperovich and A. Sopasakis, Stochastic description of traffic flow, J. Stat. Phys., 133(6), 1083-1105, 2008.

[2] A. Brandt, Multi-scale computation: from fast solvers to systematic upscaling, K.J. Bathe (ed.), Computational fluid and solid mechanics, Elsevier, 2003.

[3] N. Dundon and A. Sopasakis, Stochastic modeling and simulation of multi-lane traffic, R. Allsop, M. Bell and B. Heydecker, (eds.), Transportation and Traffic Theory, ISTTT17, 661689. Elsevier, 2007.

[4] W. E and B. Engquist, The heterogeneous multiscale method, Commun. Math. Sci., 87-133, 1(1), 2003.

[5] M.I. Friedlin and A.D. Wentzell, Random Perturbations of Dynamical Systems, Springer, 1998.

[6] M.A. Katsoulakis, A.J. Majda and A. Sopasakis, Multiscale couplings in prototype hybrid deterministic/stochastic systems: part I, deterministic closures, Commun. Math. Sci., 2(2), 255-294, 2004.

[7] M.A. Katsoulakis, A.J. Majda and A. Sopasakis, Multiscale couplings in prototype hybrid deterministic/stochastic systems: part II, stochastic closures, Commun. Math. Sci., 3(3), 453-478, 2005.

[8] M.A. Katsoulakis, A.J. Majda and A. Sopasakis, Intermittency, metastability and coarse graining for coupled deterministic-stochastic systems, Nonlinearity, 19, 1-=27, 2006.

[9] M.A. Katsoulakis, A.J. Majda, and D.G. Vlachos, Coarse-grained stochastic processes and Monte Carlo simulations in lattice systems, J. Comp. Phys., 186, 250-278, 2003.

[10] M.A. Katsoulakis, A.J. Majda and D.G. Vlachos, Coarse-grained stochastic processes for lattice systems, Proc. Natl. Acad. Sci., 100, 782-787, 2003.

[11] M.A. Katsoulakis, P. Plecháč and A. Sopasakis, Error control and analysis in coarse-graining of stochastic lattice dynamics, SIAM J. Numer. Anal., 44, 2270-2296, 2006.

[12] M.A. Katsoulakis and D.G. Vlachos, Hierarchical kinetic Monte Carlo simulations for diffusion of interacting molecules, J. Chem. Phys., 112(18), 2003.

[13] I.G. Kevrekidis, C.W. Gear, J. Hyman, P.G. Kevrekidis, O. Runborg and C. Theodoropoulos, Equation-free, coarse-grained multiscale computation: enabling microscopic simulators to perform system-level tasks, Commun. Math. Sci., 1(4), 715-762, 2003.

[14] B. Khouider, A.J. Majda and M.A. Katsoulakis, Coarse-grained stochastic models for tropical convection and climate, Proc. Natl. Acad. Sci., 100, 11941-11946, 2003.

[15] R. Lam and D.G. Vlachos, Multiscale model for epitaxial growth of films: growth mode transition, Phys. Rev. B, 64, 35401, 2001.

[16] C. Landim and C. Kipnis, Scaling Limits of Interacting Particle Systems, Springer Berlin, 1999.

[17] T.M. Liggett, Stochastic Interacting Systems: Contact Process, Voter, and Exclusion Process, Springer-Verlag, Berlin Heidelberg, 1999. 
[18] A.J. Majda and B. Khouider, Stochastic and mesoscopic models for tropical convection, Proc. Nat. Acad. Sci., 99, 1123, 2002

[19] J.D. Murray, Mathematical Biology, Springer-Verlag, 1989.

[20] S.T. Rodgers and K.F. Jensen, Multiscale modeling of chemical vapor deposition, J. Appl. Phys., 83(1), 524, 1998.

[21] A. Sopasakis, Stochastic noise approach to traffic flow modeling, Physica A, 342(3-4), 741-754, 2004.

[22] A. Sopasakis and M.A. Katsoulakis, Stochastic modeling and simulation of traffic flow: asep with arrhenius look-ahead dynamics, SIAM J. Appl. Math., 66(3), 921-944, 2006.

[23] D.G. Vlachos, L.D. Schmidt and R. Aris, The effects of phase transitions, surface diffusion and defects on surface catalyzed reactions: fluctuations and oscillations, J. Chem. Phys., 93, 8306, 1990.

[24] H.S. Wiley, S.Y. Shvartsman and D.A. Lauffenburger, Computational modeling of the egf receptor system: a paradigm for systems biology, Trends Cell Biol., 13, 43-50, 2003. 